University of Louisville

ThinkIR: The University of Louisville's Institutional Repository

$4-2019$

\title{
Experimental investigation of the manufacture of tunable graphene oxide filter membranes using intense pulse light
}

Joshua H Kotter

University of Louisville

Follow this and additional works at: https://ir.library.louisville.edu/etd

Part of the Heat Transfer, Combustion Commons, Manufacturing Commons, and the Membrane Science Commons

\section{Recommended Citation}

Kotter, Joshua $\mathrm{H}$, "Experimental investigation of the manufacture of tunable graphene oxide filter membranes using intense pulse light" (2019). Electronic Theses and Dissertations. Paper 3448.

https://doi.org/10.18297/etd/3448

This Master's Thesis is brought to you for free and open access by ThinkIR: The University of Louisville's Institutional Repository. It has been accepted for inclusion in Electronic Theses and Dissertations by an authorized administrator of ThinkIR: The University of Louisville's Institutional Repository. This title appears here courtesy of the author, who has retained all other copyrights. For more information, please contact thinkir@louisville.edu. 


\title{
EXPERIMENTAL INVESTIGATION OF THE MANUFACTURE OF TUNABLE GRAPHENE OXIDE FILTER MEMBRANES USING INTENSE PULSED LIGHT
}

\author{
By \\ Joshua Kotter \\ B. S. in Mechanical Engineering, University of Louisville, 2018
}

\begin{abstract}
A Thesis
Submitted to the Faculty of the University of Louisville

J. B. Speed School of Engineering as Partial Fulfillment of the Requirements for the Professional Degree of

MASTER OF ENGINEERING

Department of Mechanical Engineering
\end{abstract}

April 2019 

EXPERIMENTAL INVESTIGATION OF THE MANUFACTURE OF TUNABLE GRAPHENE OXIDE FILTER MEMBRANES USING INTENSE PULSED LIGHT

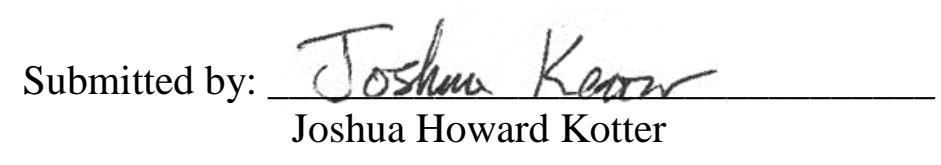

A Thesis Approved On

$\frac{05 / 01 / 2019}{\text { Date }}$

by the Following Reading and Examination Committee:

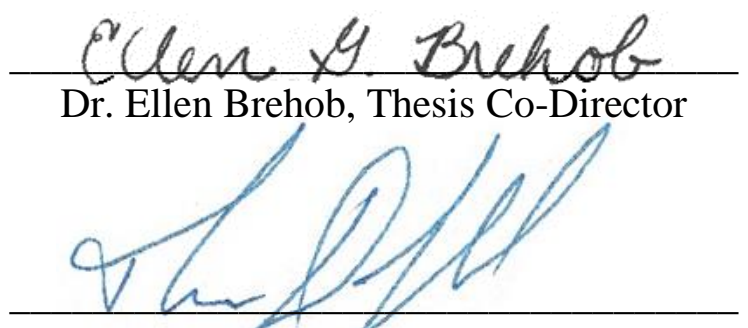

Dr. Thad Druffel, Thesis Co-Director

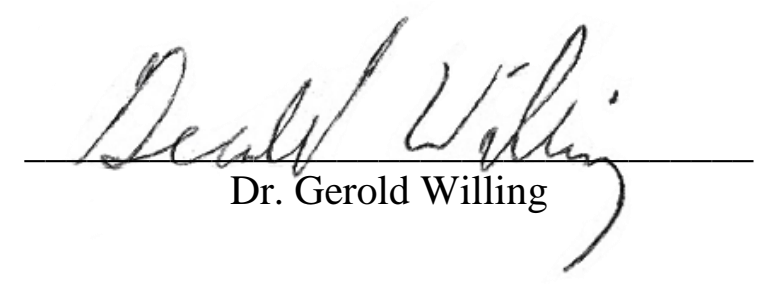




\section{ACKNOWLEDGEMENTS}

Many thanks to Dr. Brehob, my thesis advisor, for her guidance and counsel.

Thanks is also due to Dr. Druffel and his lab team for their advice, training, and help.

Funding for some of the materials for this project was provided through EPSCOR Grant \# 1355438 the generous assistance of Dr. Bhattacharyya and his team at the University of Kentucky.

Finally, I thank Jenae, the love of my life, for her love of God, gracious encouragement, and undying commitment to a joyful life together. 


\section{ABSTRACT}

Reduced graphene oxide is a thin, strong, and inexpensive material with a channel and pore structure that make it a promising candidate for a filtration material. Reduced graphene oxide has been produced and tested in the laboratory, but a lack of scalable manufacturing techniques have limited its commercial use.

This thesis has shown that graphene oxide can be rapidly manufactured with an industrially scalable flash reduction process. The flash reduction process uses 0.58 millisecond pulses from a xenon lamp to reduce the graphene oxide film in less than a minute. Results for partially reduced graphene oxide membranes tuned by the length of exposure have variable filtration flux and filtrate rejection rates. Graphene oxide films were found to reject $20 \%$ to $90 \%$ of a methyl-red dye solution, depending on their reduction level. Finally, the color of graphene oxide films was correlated to their reduction level using digital photography. Graphene oxide films were exposed to 10, 40, 70 , and 100 light pulses with xenon lamp powers of $1.8 \mathrm{kV}, 2.0 \mathrm{kV}$, and $2.2 \mathrm{kV}$. The colors of the resulting films were determined by the amount of energy the films had received.

The experimental methods used to obtain these results include vacuum filtration of graphene oxide monolayer dispersions, flash reduction of the resulting films, and pressurized filtration testing. The experimental results were characterized by atomic force microscopy, scanning electron microscopy, digital color measurement, and ultraviolet and visible light spectrophotometry. 


\section{TABLE OF CONTENTS}

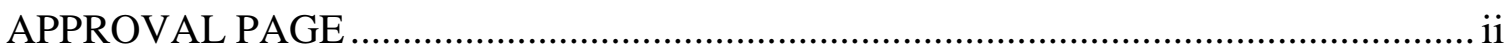

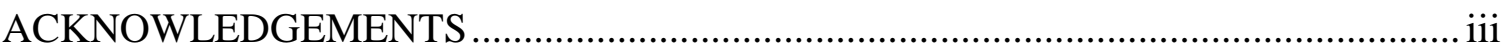

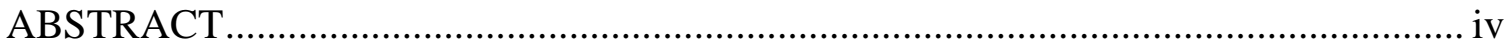

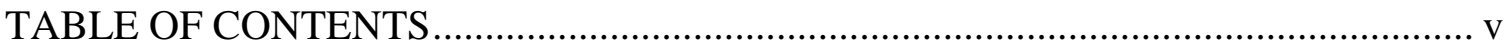

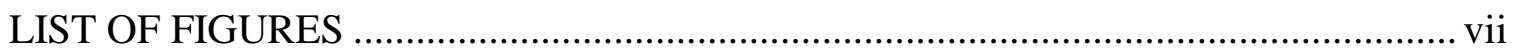

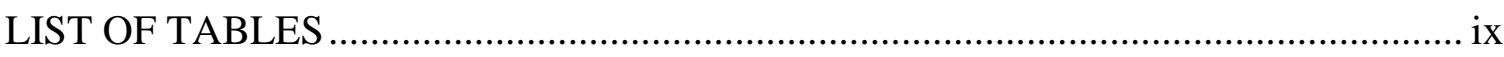

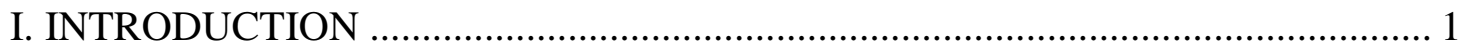

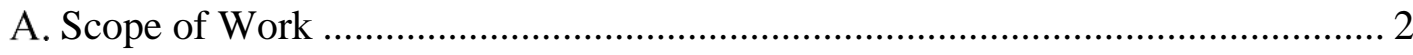

II. BACKGROUND AND LITERATURE REVIEW ………………….................. 3

A. Stages of Reduced Graphene Oxide Production .................................................... 3

B. Graphite 4

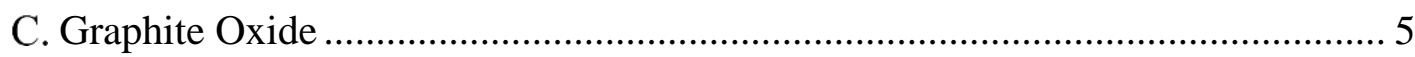

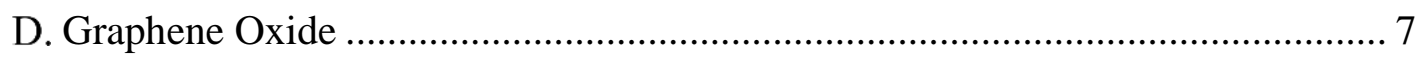

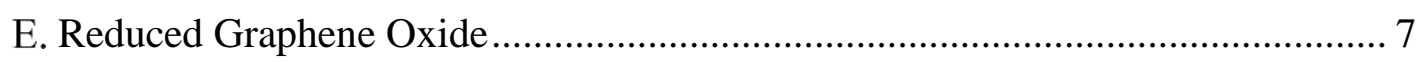

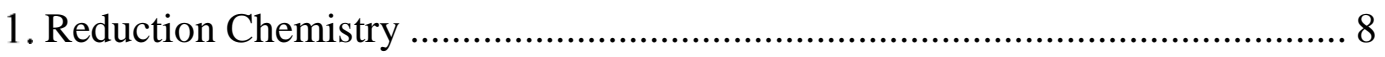

2. Manufacturing Methods............................................................................. 8

3. Applications for Reduced Graphene Oxide ..................................................... 12

4. Filtration Performance ................................................................................. 13

5. Characterization of Reduced Graphene Oxide …………………………........ 16

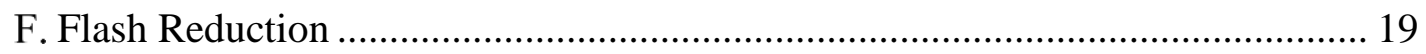

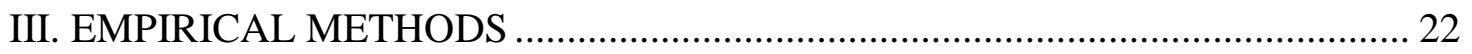

A. Sample Preparation ................................................................................. 22

1. Graphene Oxide Solution Preparation ........................................................... 22

2. Vacuum Deposition .............................................................................. 22

3. Flash Reduction ……………………………….................................. 23

4. Filtration Testing .................................................................................. 24

B. Characterization Methods ………………………......................................... 27

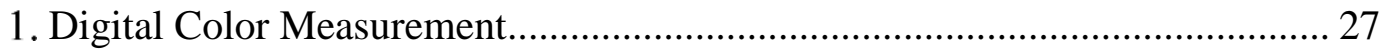

2. Scanning Electron Microscopy …………………........................................ 27

3. Atomic Force Microscopy (AFM) .............................................................. 28

4. Spectrophotometer Measurements............................................................... 28

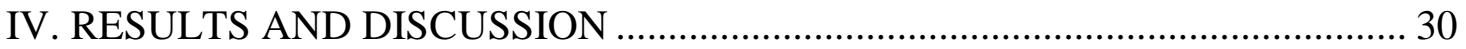




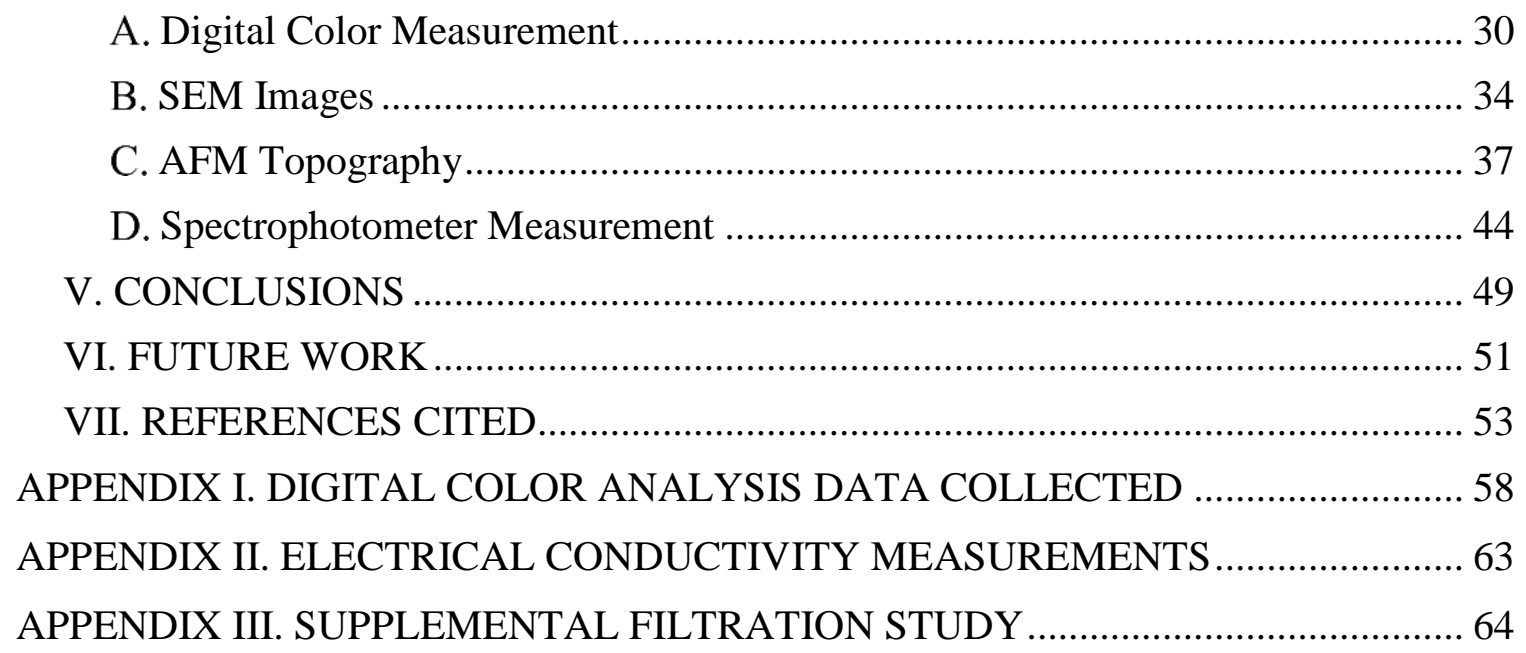




\section{LIST OF FIGURES}

FIGURE 1 - Scalable Manufacturing Method for Reduced Graphene Oxide Membranes. 1

FIGURE 2. Stages of Reduced Graphene Oxide Manufacture (Chun Li 2012)................ 3

FIGURE 3. Orbital Hybridization for Carbon Atoms in Graphite Sheet. ......................... 4

FIGURE 4. Change in Sheet Morphology and Interlayer Spacing in Graphite Oxide ....... 5

FIGURE 5. Hybridization for Bonded Oxygen Groups in Graphite Oxide. .................... 6

FIGURE 6. Tortuous Path for a Water Molecule through Graphene Oxide Layers......... 13

FIGURE 7. Reduction in Interlayer Spacing as a Result of Reduction ......................... 14

FIGURE 8. Increase in Interlayer Spacing and Decrease in Path Length. ..................... 15

FIGURE 9. Heat Transfer Boundary Conditions for Filter Substrate Model.................. 20

FIGURE 10. Vacuum Filtration Experimental Setup.............................................. 23

FIGURE 11. Pulse Energy at Various Power Levels for Xenon Lamp......................... 24

FIGURE 12. Filtration Membrane Test Cell Experiment Setup.................................. 25

FIGURE 13. As-Deposited Graphene Oxide on Glass............................................. 30

FIGURE 14. Xenon Lamp Power Setting Region for Color Analysis. ......................... 31

FIGURE 15. Graphene Oxide Films Exposed to Various Pulse Powers and Amounts. .. 32

FIGURE 16. Blue Light Intensity at Various Pulse and Power Levels......................... 33

FIGURE 17. Cross Section of Graphene Oxide Film and PVDF Filter Paper................ 34

FIGURE 18. Cross Section SEM Image of As-Deposited Graphene Oxide Film........... 35

FIGURE 19. Twelve Micron Square Scan of Untreated Polymer Substrate................... 38

FIGURE 20. Two Micron Square Scan of Untreated Polymer Substrate....................... 39

FIGURE 21. Twelve Micron Square Scan of As-Deposited Graphene Oxide Film....... 40

FIGURE 22. Two Micron Square Scan of As-Deposited Graphene Oxide. ................... 41 
FIGURE 23. Twelve Micron Square Scan of Reduced Graphene Oxide Film.

FIGURE 24. Two Micron Scan of Reduced Graphene Oxide Film............................. 43

FIGURE 25. Graphene Oxide Samples Prepared for Filtration Testing. ........................ 44

FIGURE 26. Filtered Solution Absorbance for Visible and Ultraviolet Spectra.............. 46

FIGURE 27. Flux and Dye Rejection for Reduced Graphene Oxide Membranes. .......... 48

FIGURE 28. Filtered Solution Absorbance for 1.8 kV Pulsed Graphene Oxide. ............ 64 


\section{LIST OF TABLES}

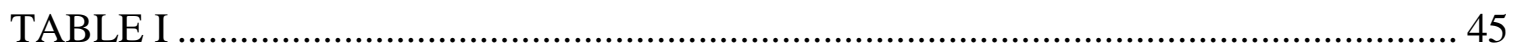

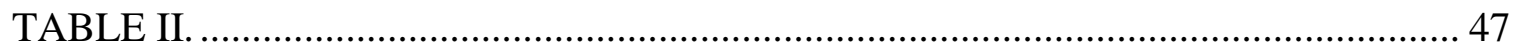

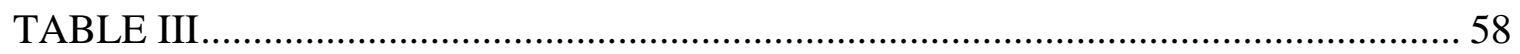

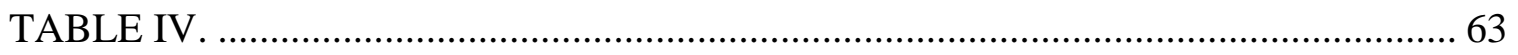

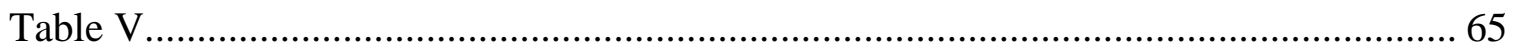

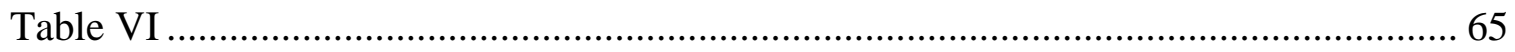




\section{INTRODUCTION}

Reduced graphene oxide is a promising thin-film material with high thermal and electrical conductivity, high strength, and extremely high surface area. Because of its tunable channel and pore structure, it is an attractive material for membrane filtration.

One of the major barriers to the commercial use of reduced graphene oxide is the long cure times and harsh chemicals necessary during the reduction process.

Additionally, the high temperatures and strong solvents involved prevent reduced graphene oxide from being reacted in-situ on many substrates, especially polymers.

The proposed method for manufacturing reduced graphene oxide sheets in a commercially scalable manner is through a roll-to-roll process diagramed in FIGURE 1.

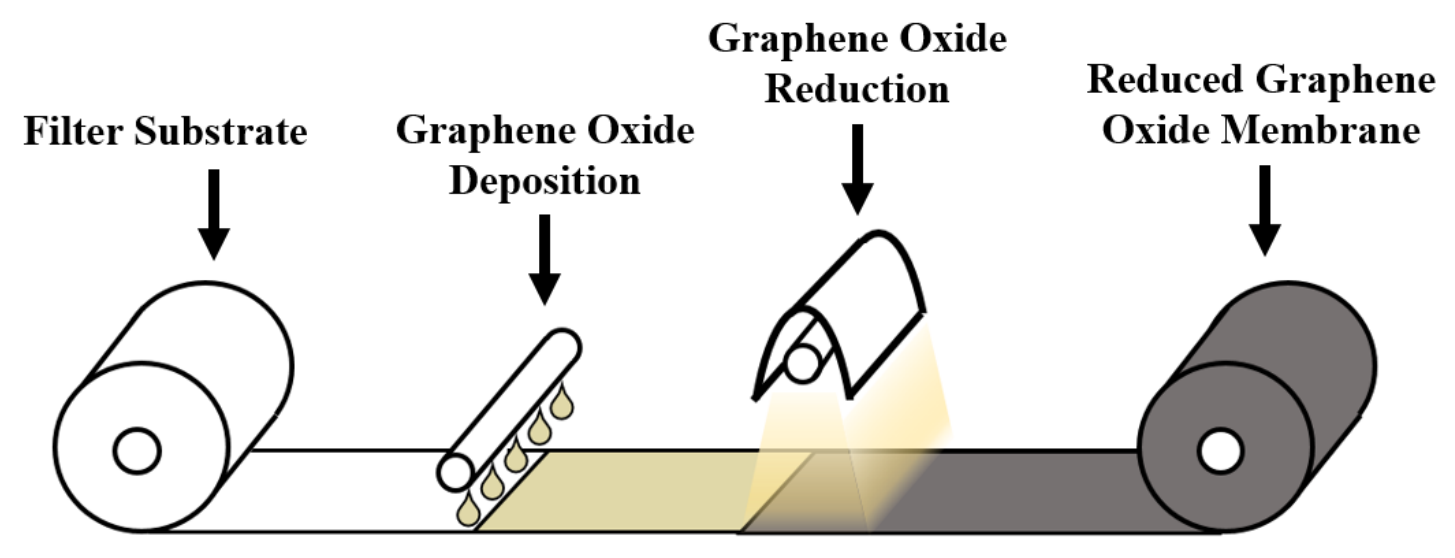

FIGURE 1 - Scalable Manufacturing Method for Reduced Graphene Oxide Membranes.

The above process can be performed on a meter-wide roll of polymer filtration membrane. This polymer layer supports the graphene oxide film and facilitates integration into existing filtration technologies. Graphene oxide can be deposited onto the substrate as a dispersion in water. After the water has dried, the resulting graphene oxide 
film can be reduced by one or more pulses of light from a xenon or other ultraviolet lamp. The resulting filter material can be produced at a commercially viable rate of square meters per minute with this industrially scalable process.

\section{A. Scope of Work}

The purpose of this thesis is to quantify and characterize a flash reduction process for reducing graphene oxide deposited on a polymer membrane. Flash reduction has been used with limited success on free-standing graphene oxide papers (Pei 2012), but it has not been considered as a way to incrementally control the reduction of as-deposited graphene oxide on polymer substrates. The process of controlling the reduction of graphene oxide on a polymer substrate opens new possibilities for the use of graphene oxide as a low-cost filtration membrane for both liquids and gases.

In order to determine the effectiveness of flash-reduced graphene oxide films as water filtration membranes, graphene oxide films were manufactured, reduced, and characterized. Graphene oxide flakes were purchased as a dispersion in water and then deposited on a cellulose-backed polymer matrix with a vacuum filtration method. After drying, these samples were exposed to light pulses from a xenon lamp at varying power settings and for different numbers of pulses.

The thickness and topography of the film before reduction was determined by AFM topography measurements and SEM cross-section scans. The level of reduction in the samples was characterized through optical color measurements and additional AFM measurements. Finally, the permeability of the samples to water and methyl-red dye was measured with a filter test cell. 


\section{BACKGROUND AND LITERATURE REVIEW}

\section{A. Stages of Reduced Graphene Oxide Production}

The manufacture of reduced graphene oxide can be broken down into four stages, as seen in FIGURE 2. Graphite can be oxidized to form graphite oxide, which in turn can be exfoliated in water into monolayer flakes called graphene oxide.

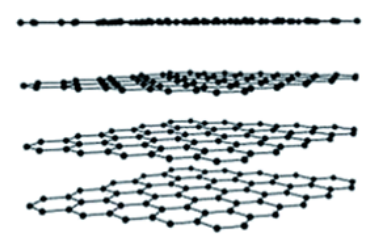

Graphite

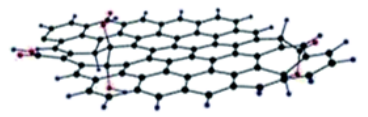

Reduced Graphene Oxide

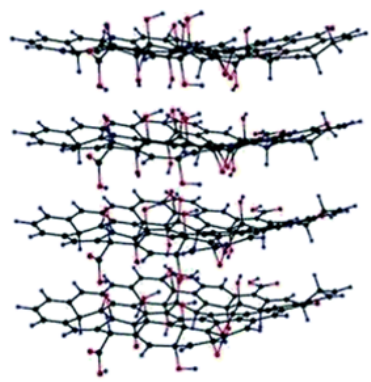

Graphite Oxide
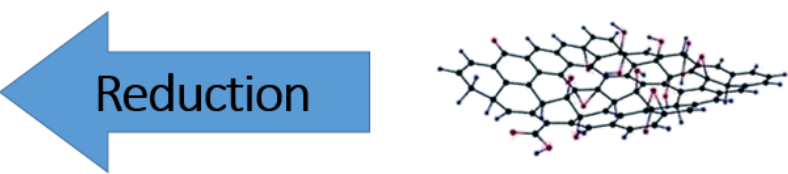

Graphene Oxide

FIGURE 2. Stages of Reduced Graphene Oxide Manufacture (Chun Li 2012).

These flakes can be reacted in a reduction process to make reduced graphene oxide. The individual stages of this process are detailed in the following sections. This thesis focuses on the reduction stage of the process, so the material used was purchased as prepared monolayer graphene oxide flakes. 


\section{B. Graphite}

Graphite is a crystalline elemental form of carbon. In graphite, carbon atoms are arranged in hexagonal-matrix sheets, as seen in FIGURE 2. Each carbon atom is covalently bonded with three other carbon atoms in $\mathrm{sp}^{2}$ hybridized orbitals (Hunt 2019), as in FIGURE 3.

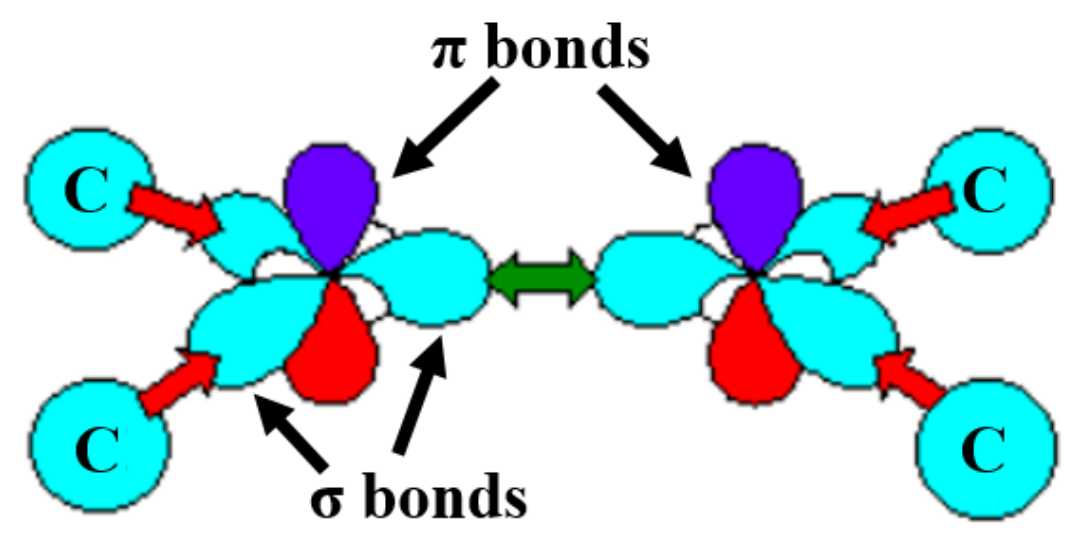

FIGURE 3. Orbital Hybridization for Carbon Atoms in Graphite Sheet.

All three $\sigma$-bonds lie in the same plane (Hunt 2019), allowing the carbon atoms to form the large hexagonal sheets of graphite visible in FIGURE 2 . The $\pi$-bonds are actually much larger than shown in FIGURE 3, and are dissociated across multiple carbon atoms. These widely dissociated $\pi$-bonds allow for low-resistance electron transport in-plane, causing graphite to be electrically conductive (Kinchin 1953). The $\pi$ bonds do not covalently link vertically between sheets. Instead, the sheets are stacked via van der Waal's forces to form the bulk structure of graphite (Kinchin 1953). 


\section{Graphite Oxide}

In the presence of strong oxidizers, oxygen atoms can intercalate between the layers of carbon atoms and bond in clusters on the graphite sheets (Compton 2010). The addition of oxygen atoms distorts the flat shape of carbon sheets and causes the sheets to be spaced approximately twice as far apart (Compton 2010). FIGURE 4 (a detail from FIGURE 2) shows the addition of wrinkles in the carbon layers and the increased space between layers caused by the oxygen groups.
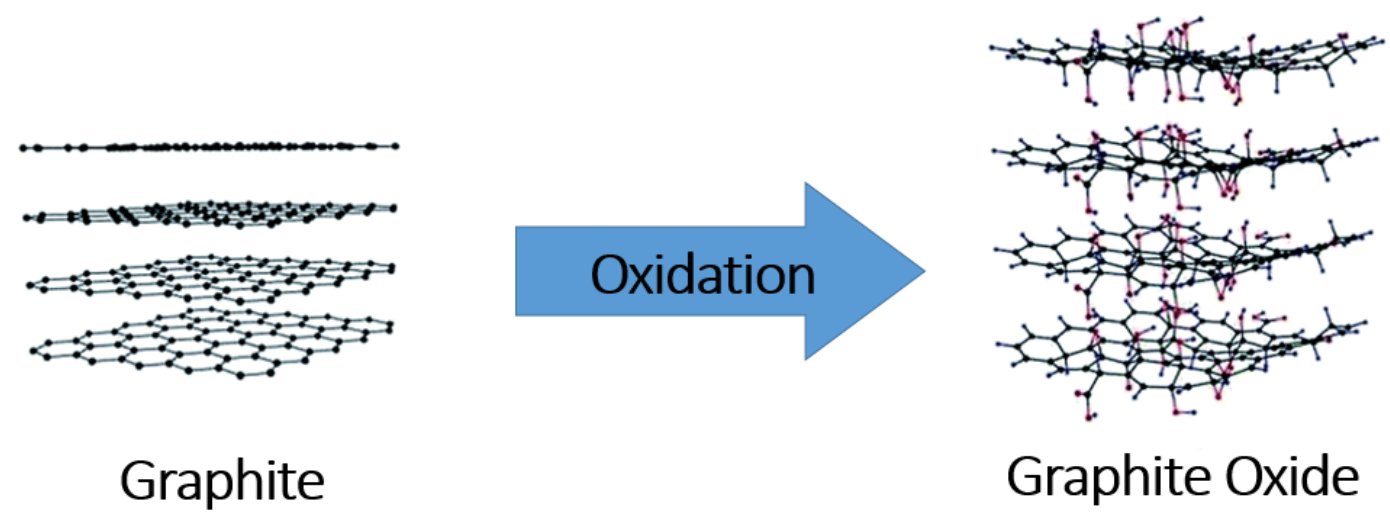

FIGURE 4. Change in Sheet Morphology and Interlayer Spacing in Graphite Oxide

The oxygen atoms bonded to the carbon sheets cause the carbon atoms to transition to a sp $\mathrm{s}^{3}$ hybridized orbital state, as in FIGURE 5. In $\mathrm{sp}^{3}$ hybridized orbitals, the $\sigma$-bonds do not all share a common plane (Hunt 2019). Groups of these out-of-plane bonds introduce wrinkles into the sheets of graphite oxide (Pei 2012). 


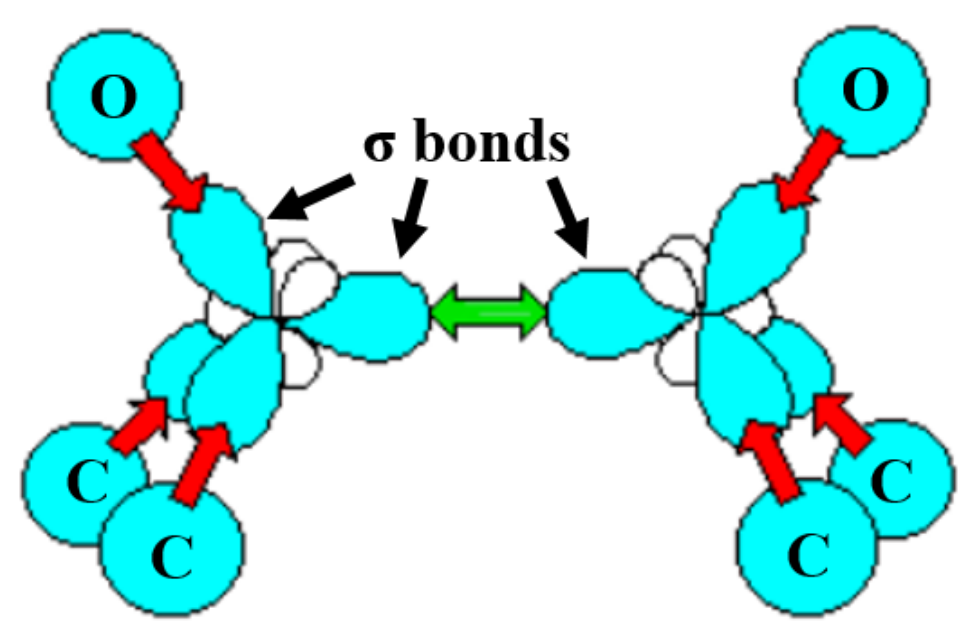

FIGURE 5. Hybridization for Bonded Oxygen Groups in Graphite Oxide.

In the covalent bonds between oxygen and carbon atoms, the oxygen atoms are more electronegative and hold a preferential share of the electrons in the $\sigma$-bond. The uneven distribution of electrons causes the oxygen atoms to be negatively charged (Compton 2010). When the graphite oxide is immersed in water, the charged oxygen groups attract the polar water molecules. The polar attraction, combined with the increased interlayer spacing, allows water molecules to intercalate between the layers of the graphite oxide (Thomas 2013). 


\section{Graphene Oxide}

Once water molecules have moved between the layers of graphite oxide, the layers can be separated with stirring or sonication (Pei 2012). These methods provide enough energy to overcome the van der Waal's between the sheets of graphite oxide and break the sheets off into individual monolayer flakes. The process of separating the atomic layers is called exfoliation. Graphene oxide flakes are 1-5 $\mu \mathrm{m}$ in diameter and 10$100 \mathrm{~nm}$ thick (Stobinski 2014). Some of the flakes are one atomic layer thick, while other flakes still consist of multiple layers. The graphene oxide used in this study was composed of over $95 \%$ monolayer flakes (Graphenea 2017). These flakes are called graphene oxide because their characteristics as flakes are significantly different from bulk graphite oxide, even though they have not experienced a chemical reaction.

\section{E. Reduced Graphene Oxide}

In order to give the graphene oxide film specific filtration characteristics, the flakes can be reduced via chemical processes, exposure to plasma or intense light, as well as through direct heating. The heating or chemical treatment of the graphene oxide flakes provides energy to break the bonds between the oxygen groups and carbon atoms. As the oxygen groups escape, the carbon atoms return to a $\mathrm{sp}^{2}$ hybridized state, resulting in reduced graphene oxide. Due to the removal of oxygen groups, reduced graphene oxide has smaller interlayer spacing than graphene oxide (Stobinski 2014). Not all of the oxygen groups can be perfectly removed, and some remaining wrinkles and defects caused by the removal of oxygen groups affect the morphology of the graphene oxide flakes (Pei 2012). 


\section{Reduction Chemistry}

A reduction reaction is one that results in an atom gaining electrons (Cortes 2010). In the case of graphene oxide, electrons are shared preferentially by oxygen atoms. When the oxygen groups are removed, the electrons are restored to the carbon atoms in a reduction reaction. Reduction is important for graphene oxide filter membranes because it controls the size of pores and defects in the reduced graphene oxide. These defects can be sized to selectively filter certain molecular compounds (R. K. Joshi 2014).

If graphene oxide is provided with sufficient energy through chemical or thermal methods under ambient atmospheric conditions, both reduction and oxidation reactions occur (Tegou 2016). Epoxy and hydroxyl groups are eliminated from the film with sufficient heating. However, in the presence of oxygen, new carbonyl groups form due to re-oxidation of the film (Pei 2012). Despite the newly formed oxygen groups, the carbon atoms in reduced graphene oxide are largely restored to $\mathrm{sp}^{2}$ hybridization from the $\mathrm{sp}^{3}$ hybridization of the original graphene oxide. The return to $\mathrm{sp}^{2}$ hybridization greatly increases the electrical and thermal conductivity of the film (Pei 2012).

\section{Manufacturing Methods}

a. Chemical Reduction. Various reducing agents have been used to obtain reduced graphene oxide from a graphene oxide dispersion. These methods are often combined with elevated temperatures to decrease the time necessary for the reaction.

One reduction agent is hydrazine hydrate. Graphene oxide dissolved in hydrazine and maintained at $100{ }^{\circ} \mathrm{C}$ for 24 hours yields evenly reduced product (Pei 2012). A film 
of graphene oxide can also be exposed to hydrazine vapor to achieve a similar reaction (Gensheimer 2015).

A more recently used reducing agent is hydroiodic acid. Like hydrazine, hydroiodic acid can be used to reduce graphene oxide in a film-vapor or solution environment (Pei 2012). Some advantages of hydroiodic acid are that it yields higher levels of reduction, can be used at room temperature, and it produces a more flexible reduced graphene oxide film (Lowe 2016).

Many other compounds have been used to achieve reduced graphene oxide, including ascorbic acid, urea, metal hydrides, and strong alkaline solutions, though with lower quality results (Yeh 2014) (Zhang 2010). Some of the limitations of chemical reduction are the length of the many-hour cure times, the difficulty in processing commercial amounts of solutions or film-vapor environments, and safety concerns with the toxic and highly reactive chemicals used (Gensheimer 2015).

b. Reduction through Thermal Annealing. Thermal reduction is another method that has been used to reduce graphene oxide. Graphene oxide deposited on a substrate or formed into a paper is reduced at around $1000{ }^{\circ} \mathrm{C}$ in ambient air and inert atmospheres (Pei 2012). Reactive atmospheres of hydrogen and ammonia have also been used, and reduction can be achieved at lower temperatures (around $500{ }^{\circ} \mathrm{C}$ ) in these conditions (Lowe 2016). Reduction in air results in some re-oxidation of carbonyl groups after other oxygen groups have been eliminated. Significant changes in conductivity can be achieved through reduction in air, though less than under inert atmospheres (Pei 2012). The reoxidation of graphene oxide has also been observed to result in more mass lost to carbon dioxide and smaller reduced graphene oxide flake sizes (Pei 2012). 
Solution-dispersed graphene oxide can also be thermally reduced. Graphene oxide dispersed in various solvents has been reduced at temperatures ranging from $500{ }^{\circ} \mathrm{C}$ to $1100{ }^{\circ} \mathrm{C}$ (Pei 2012), with higher temperatures resulting in increased levels of reduction. Certain solvents cannot be used for this type of reduction due to their low flash point. The oxidation of these solvents can be controlled with an inert atmosphere, but this additional step adds cost and complexity to the reduction process (Pei 2012).

One of the major drawbacks of thermal annealing is its high energy consumption. Another limitation is the choice of substrates. Due to the high temperatures involved, graphene oxide films on polymer substrates cannot be reduced with this method. Instead, graphene oxide flakes must be reduced while dispersed in water and then deposited on a substrate. Since reduced graphene oxide is harder to disperse in water due to its reduced number of oxygen groups, additional chemicals must be added to allow the reduced graphene oxide to be deposited evenly (Azizighannad 2018).

c. Electrochemical Reduction. An emerging method for reducing graphene oxide are electrochemical methods. In this process, the film is placed in an aqueous solution opposite an inert electrode. As the electrochemical cell is charged, some reduction of the film occurs (Pei 2012). The reduction process in this situation is not fully understood, although the leading hypothesis is that $\mathrm{H}^{+}$ions interact with the graphene oxide during cell charging. This method does not require harsh chemicals and can be executed at room temperature, giving it some advantages over chemical reduction. However, only relatively low-conductivity films of reduced graphene oxide have been produced up to this point (Pei 2012). 
d. Flash Reduction. As an alternative to traditional thermal irradiation, highenergy light sources have been used to quickly heat and reduce graphene oxide. Microwaves, xenon lamps, and lasers fall into this category. These methods provide even and rapid heating of the graphene oxide surface.

Microwaving a graphite oxide powder can yield reduced graphene oxide in less than a minute. The rapid expansion of gases trapped between layers of the graphite oxide cause mechanical exfoliation of the graphite oxide even as the reduction process takes place (Pei 2012). While this simplifies the steps needed to obtain graphene oxide, a significant amount of mass is lost to carbon dioxide production (Gensheimer 2015).

A focused laser beam has been used to irradiate graphene oxide with femtosecond pulses. This resulted in a reduced graphene oxide with a higher conductivity than other photo reduction methods. Use of the laser also allowed pre-programmed conductive patterns to be drawn on the insulating graphene oxide layer (Pei 2012). This is useful for small scale patterning, but is difficult to scale up to large areas of reduced graphene oxide.

Finally, a xenon flash lamp can be used to reduce graphene oxide films that are deposited on a substrate or free standing (Pei 2017). Distance to the lamp and pulse power can both be varied to control the amount of reduction accomplished by the lamp. In a process similar to microwaving, the layers of graphene oxide tend to expand during reduction due to outgassing (Pei 2012).

Flash reduction has the potential to produce reduced graphene oxide films on a polymer substrate in short period of time (Pei 2017). This is a significant advantage over thermal annealing using conventional methods, which require hours or days to complete. 
It is also safer and more versatile than chemical methods, which are generally not compatible with polymers.

\section{Applications for Reduced Graphene Oxide}

Reduced graphene oxide was originally investigated as a route to the production of graphene. Pristine graphene has extremely high thermal and electrical conductivity, as well as high strength and near transparency (Khan 2016). However, reduced graphene oxide has residual oxygen groups and lattice defects that keep it from having the same conductivity as graphene. Researchers are investigating methods of healing these defects to gain graphene-like properties from reduced graphene oxide (Pei 2012).

Other applications of graphene oxide and reduced graphene oxide stem from the contrast in their properties. Unreacted graphene oxide is an insulator, while reduced graphene oxide is a conductor. This has led researchers to reduce portions of a graphene oxide film to create thin and flexible printed circuits and electronics (Huang 2011). Reduced graphene oxide has also been used as a key component of conductive inks, allowing circuits to be printed on numerous substrates (Pei 2012).

Because of its light weight and high mechanical strength, graphene oxide has been used in a number of composites. It has been made into graphene rubbers with increased durability and heat dissipation (Lowe 2016). It has been used to add hydrophilic and antifouling qualities to polymer ultrafiltration materials (Huang 2011). Reduced graphene oxide is also being researched as a composite material in lithium-ion battery cathodes (Pei 2012). 


\section{Filtration Performance}

The filtration performance of graphene oxide membranes is an effect of the layered structure of a graphene oxide film. When graphene oxide flakes are deposited on a surface, the thin flakes align and form a layered membrane (Long Chen 2018). Spaces between flakes and defects in the surface of the flakes allow water and dissolved materials to pass through and between the layers in a tortuous path, as in FIGURE 6.

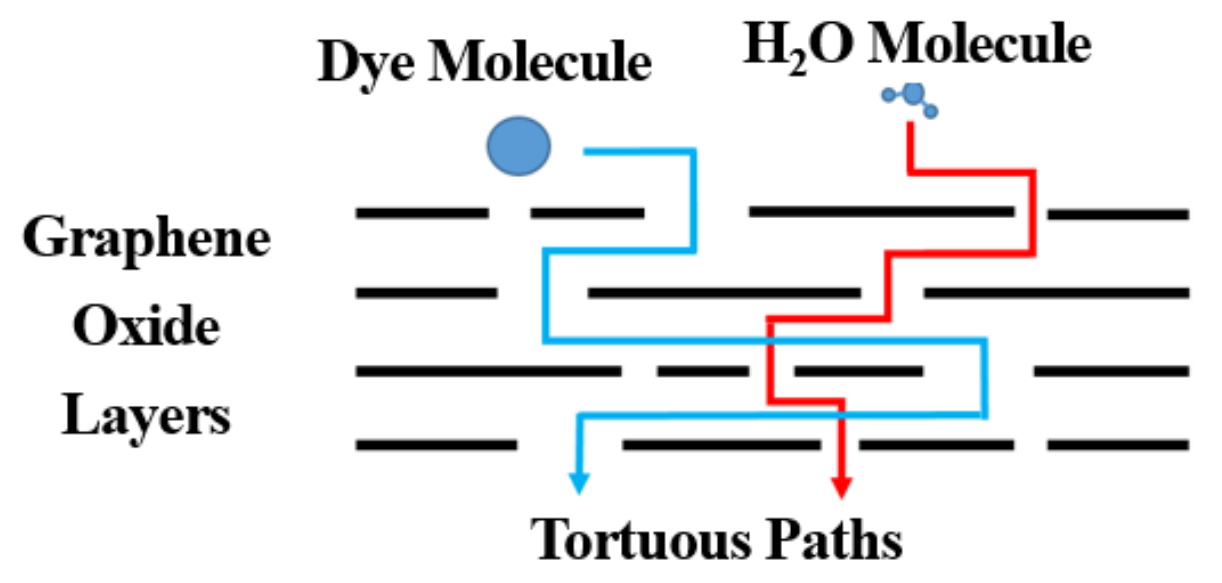

FIGURE 6. Tortuous Path for a Water Molecule through Graphene Oxide Layers.

The size of molecule that is permitted through the membrane is determined by the size of defects in each layer of the membrane, as well as the interlayer spacing between flakes. During the reduction process, a graphene oxide film changes in two ways. First, due to the removal of oxygen atoms from the flakes, the flakes become thinner and can nest more closely together, as in FIGURE 7. The decrease in interlayer spacing serves to reduce the size of particles that are allowed through the membrane (Wanbin Li 2018). 

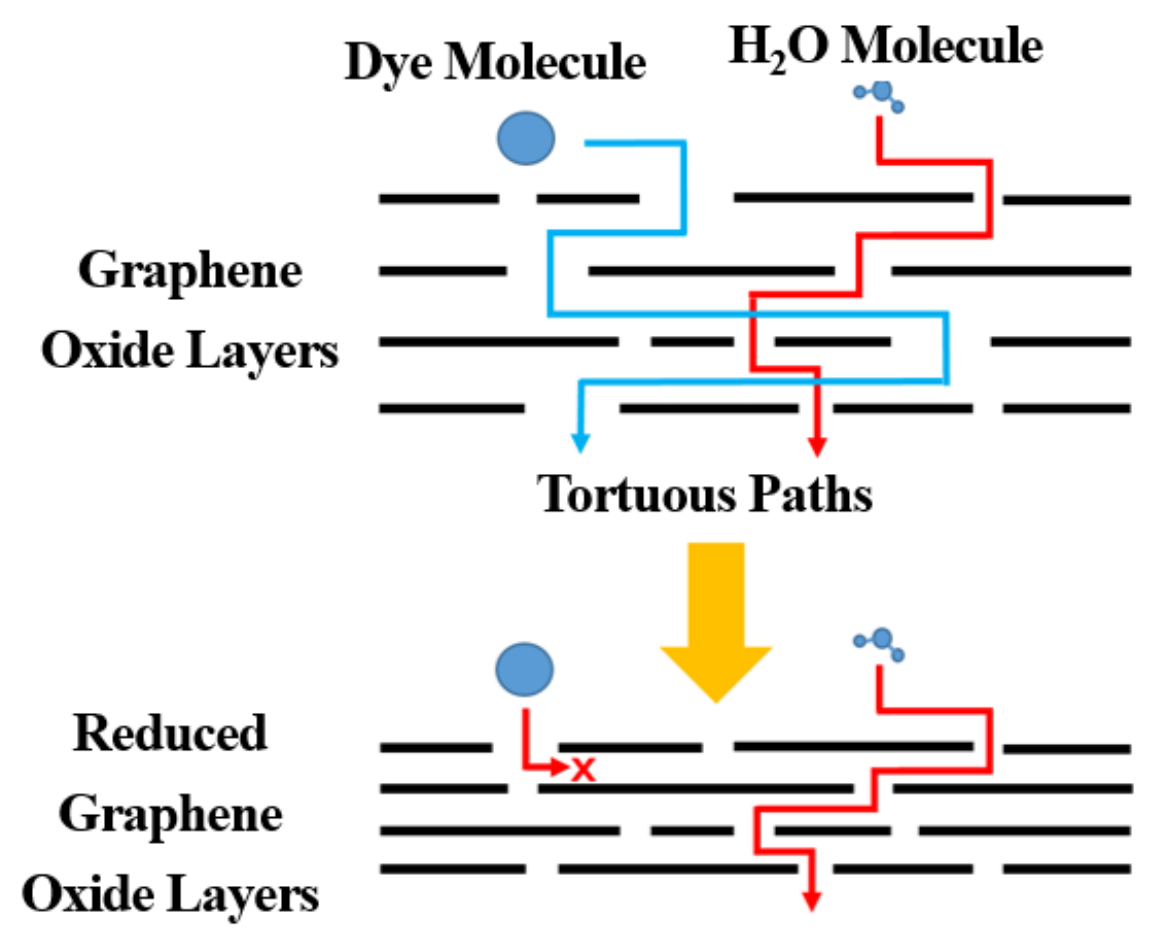

FIGURE 7. Reduction in Interlayer Spacing as a Result of Reduction

The second change that occurs in a graphene oxide membrane during the reduction process is an increase in defects and wrinkles in the graphene oxide flakes. As oxygen groups are removed from the flake surface, they leave behind holes, distortions, other defects in the flakes. Additionally, the freed oxygen groups form small pockets of gas between the layers of graphene oxide. As this gas heats and expands, it can cause additional defects by tearing through the graphene oxide layers and escaping in a process called outgassing (Pei 2012). The curling and distortion of flakes increases the interlayer spacing of the membrane and creates more channels for water and particles to pass through the membrane, as in FIGURE 8. 


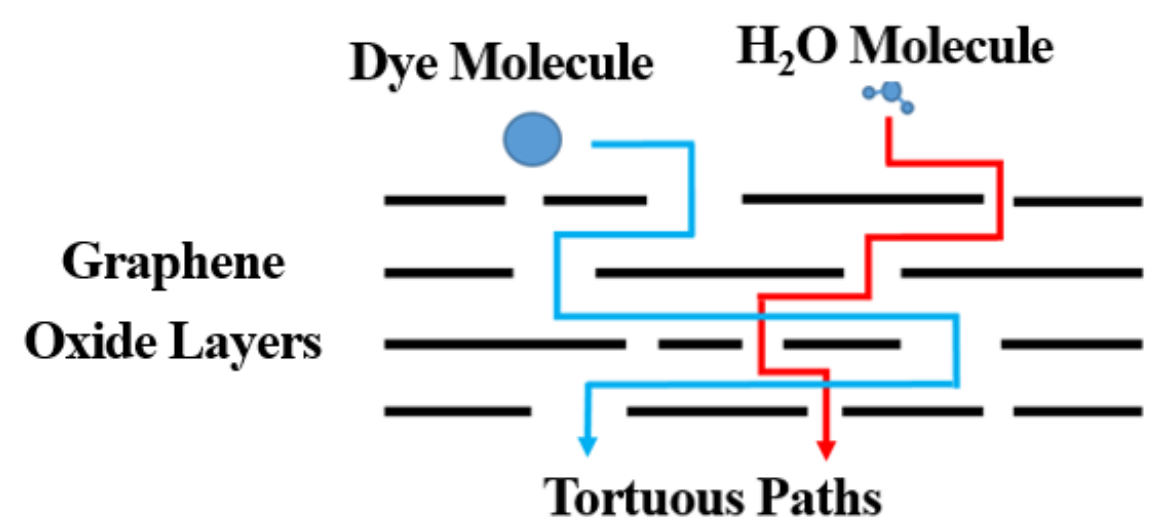

\section{Reduced \\ Graphene \\ Oxide Layers}

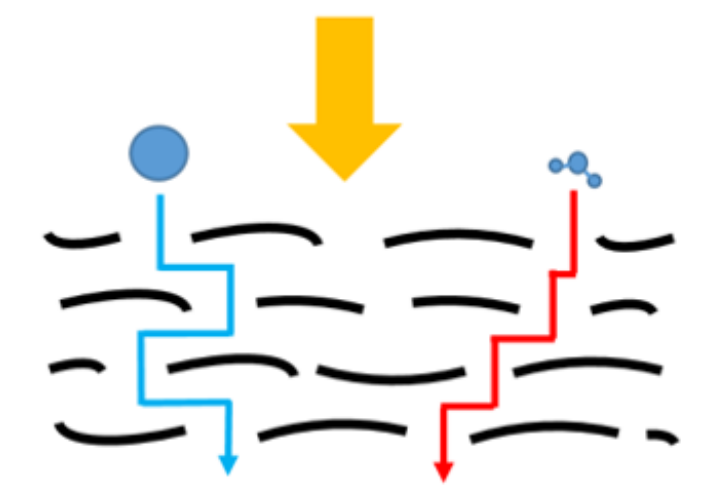

FIGURE 8. Increase in Interlayer Spacing and Decrease in Path Length.

The change in flake thickness due to the removal of oxygen and the distortion of flakes during reduction have opposing effects on the final interlayer spacing of the reduced graphene oxide membrane. Different reduction techniques can allow one effect to dominate the final film characteristics. Thermal annealing and chemical reduction are slower processes that reduce interlayer spacing while minimizing film distortion. By contrast, the short reaction times and speed of temperature changes in flash reduction tends to produce more distorted flakes (Pei 2012) with wider interlayer spacing. 


\section{Characterization of Reduced Graphene Oxide}

a. Mechanical Testing. Graphene oxide can be characterized by a variety of techniques, including mechanical, electrical, and elemental analysis. Mechanical techniques include tensile and bending tests of freestanding graphene oxide papers. Tests have found that graphene oxide paper has a very high elastic modulus and tensile strength for carbon-based papers (Dikin 2007). Interestingly, the water content of the graphene oxide significantly affected its mechanical properties. Slightly wet graphene oxide paper had a lower elastic modulus and tensile strength than dry samples, in a manner similar to cellulose paper (Dikin 2007).

Another method of testing individual graphene oxide flakes is through atomic force microscopy. This was accomplished by depositing monolayer graphene oxide flakes over a holey carbon surface. When a clear, unwrinkled flake spanned one of the well-defined circular holes, an AFM probe could be used to apply small forces and measure displacements (Suk 2010). AFM can also be used to gain surface topography information about graphene oxide films and changes that occur when the film is reduced.

b. Electrical Testing. Electrical techniques include 4-point-probe conductivity measurements of bulk films (Eluyemi 2016) as well as various types of electron microscopy (Zunita 2018). Conductivity measurements are a useful proxy for measuring a graphene oxide film's reduction state. The $\pi$-bonds in $\mathrm{sp}^{2}$ hybridized carbon materials largely control the material's optical and electrical characteristics. Graphene oxide includes large clusters of $\mathrm{sp}^{3}$ hybridized carbon atoms due to the oxygen groups decorating the carbon atom plane (Pei 2012). 
As the graphene oxide is reduced, many of the carbon atoms are returned to an $\mathrm{sp}^{2}$ hybridized state, resulting in an increase in charge carriers in the basal plane. The increase in charge carriers greatly increases conductivity (up to seven orders of magnitude were observed in measurements from this study listed in Appendix II) of the reduced graphene oxide. The additional charge carriers also improve the reflectivity of incident visible light, causing the material to gain a metallic luster (Pei 2012). This contrasts sharply with the original light-brown, semi-transparent graphene oxide (Pei 2012).

In addition to changing the optical characteristics of graphene oxide, the conversion of carbon hybridization from $\mathrm{sp}^{3}$ to $\mathrm{sp}^{2}$ orbitals greatly increases the electrical conductivity of reduced graphene oxide (Compton 2010). Although reduced graphene oxide includes more defects in the atomic plane and more oxygen groups than pristine graphene, it has similar, if somewhat reduced electrical conductivity. While graphene oxide is a mild insulator because of its predominantly $\mathrm{sp}^{3}$ hybridization, reduced graphene oxide displays increased electrical conductivity with increased levels of reduction (Compton 2010).

c. Elemental Analysis. The elemental makeup and bond structure of graphene oxide films can be measured with Raman spectroscopy (Wu 2018), infrared spectroscopy (Zhang 2010), X-ray photoelectron spectroscopy (XPS), and X-ray diffraction (Stobinski 2014). These methods bombard a graphene oxide film with various forms of radiation and measure the reflected radiation or freed electrons.

XPS analysis is particularly interesting because it gives information about the elements present and the ways that the atoms are bonded. The average number of oxygen 
group decorations, as well as the type of group, can be gleaned from this analysis. The particular spectrum of interest is electrons freed from the 1s orbital of carbon atoms $(\mathrm{C} 1 \mathrm{~s})$ by X-rays. A $\mathrm{sp}^{2}$ hybridized carbon atom bonded with other carbon atoms has a distinctive C1s binding energy of $284.8 \mathrm{eV}$ (Stobinski 2014). By contrast, $\mathrm{sp}^{3}$ and $\mathrm{sp}^{2}$ bonds with oxygen groups are measured at separate energy peaks. As a graphene oxide film is reduced, the oxygen peaks decrease in magnitude and the $\mathrm{C}-\mathrm{C}$ peak increases (Stobinski 2014).

X-ray diffraction gives useful information about the interlayer spacing in bulk graphene oxide films. By shining X-rays through thin stacks of graphene oxide flakes and measuring the resulting changes in angle, the space between graphene oxide sheets can be measured (Stobinski 2014). This spacing has been observed to vary with humidity and moisture content of the flakes, as water molecules intercalate between the layers. 


\section{F. Flash Reduction}

As mentioned previously, flash reduction is the use of high energy light pulses to reduce graphene oxide. Flash reduction was the focus of this investigation due some of its unique properties. It is a very fast process, using light pulses that last less than a second (Williams 2010). Additionally, the light is absorbed in the graphene oxide layer, providing localized heating to the graphene oxide with minimal damage to the substrate (Druffel, Dharmadasa et al. 2018).

To predict the temperatures that would be present in the sample during the flash reduction process, an analytical model was constructed using heat transfer principles. This section will detail the mathematical model and assumptions used in the analytical model.

The sample was approximated as an infinitely large plate, neglecting edge effects. The top layer of the plate was exposed to ambient air. The bottom of the sample lightly rested on a glass microscope slide. Because the sample did not have good thermal contact with the glass, the paper-air-glass interface was approximated as insulated. The boundary conditions are illustrated in FIGURE 9.

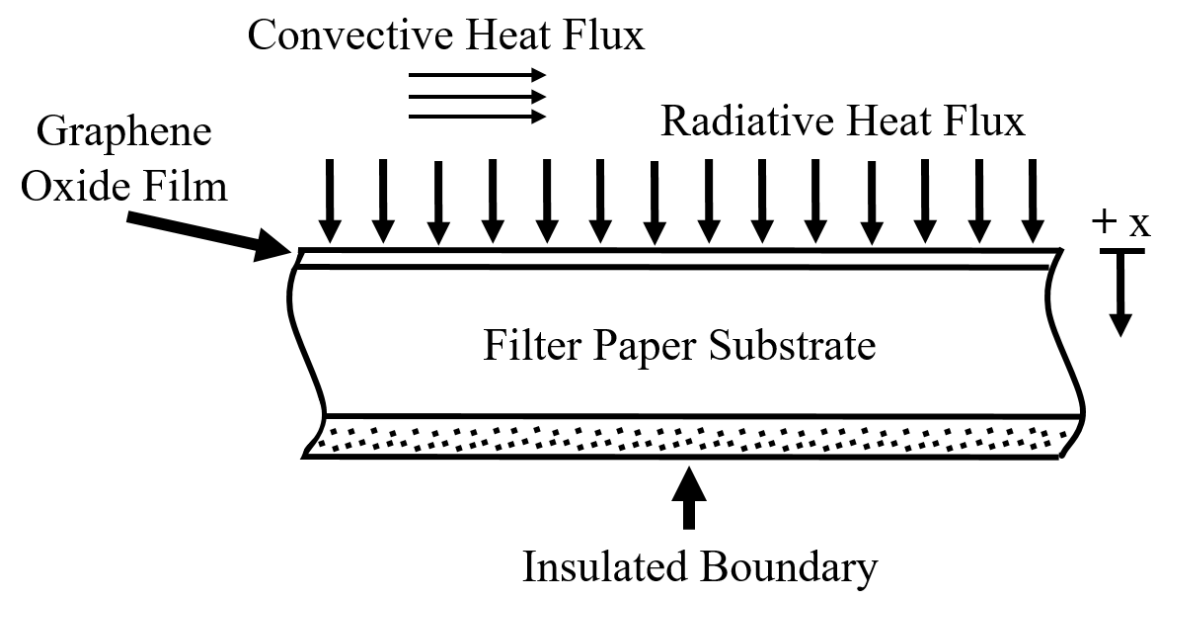


FIGURE 9. Heat Transfer Boundary Conditions for Filter Substrate Model.

The graphene oxide layer was measured to be around half a percent of the total thickness of the sample. Since the graphene oxide layer was so small relative to the paper, it was neglected at this stage in the model.

The transient temperature through the sample was modelled using 1-D conduction for a semi-infinite solid with an energy pulse at the surface (Çengel 2015) as

$$
T(x, t)-T_{i}=\frac{e_{s}}{k \sqrt{\frac{\pi t}{\alpha}}} \exp \left(-\frac{x^{2}}{4 \alpha t}\right)
$$

where $T_{i}$ is the initial temperature, $x$ is the distance from the surface, $t$ is the time elapsed, $e_{s}$ is the heat flux present at the surface during the energy pulse, $k$ is the thermal conductivity, and $\alpha$ is the thermal diffusivity of the sample.

The radiant heat flux term, $e_{s}$, was crucially important to the model. The radiant heat flux absorbed by the graphene oxide was a function of the absorption length of the ultraviolet light that was incident on the film. Since the graphene oxide film was partially transparent, any light that was transmitted through the film or that was reflected did not contribute to the heating of the graphene oxide. Additionally, the graphene oxide changed color and becomes more opaque as it reduced, especially in the high-energy ultraviolet spectrum, so it tended to heat more quickly as it reduced. The xenon lamp used to provide the heat flux converted at least $30 \%$ of its electrical pulse to optical radiation, with a nominal range of $1-30 \mathrm{~J} / \mathrm{cm}^{2}$ per pulse incident on the graphene oxide film.

The model also assumes constant and homogenous thermal properties, and that a uniform heat flux is applied to the surface. Finally, the equation assumes the analysis takes place over a short enough time frame that the heat does not have time to penetrate 
completely through the sample, allowing the sample to be approximated as a semiinfinite solid.

The heat distribution described by Equation 1 is hottest at the surface exposed to radiation. The temperature change due to the radiation heating decays exponentially at deeper distances into the paper. 


\title{
III. EMPIRICAL METHODS
}

\author{
A. Sample Preparation
}

\section{Graphene Oxide Solution Preparation}

The graphene oxide for the experiments was prepared using the Hummers method. The graphene oxide was purchased as a highly concentrated ( $25 \mathrm{~g} / \mathrm{L})$ dispersion from Graphenea. The concentrated graphene oxide was diluted with distilled water at a concentration of $1 \mathrm{mg} / \mathrm{mL}$ and sonicated on low power for 15 minutes with a Branson 1510 ultrasonic cleaner. The solution was prepared in bulk $(250 \mathrm{~mL})$ and sonicated for 10 minutes each day before use.

\section{Vacuum Deposition}

The graphene oxide was deposited on a PV200 PVDF filtration membrane with a $50 \mathrm{~nm}$ pore size using a vacuum filtration process. The substrate was purchased in sheets and cut to size in $25 \mathrm{~mm}$ circles. The substrate was pre-wet on a sintered glass Büchner funnel. As shown in FIGURE 10, the Büchner funnel was set in a Büchner flask. 


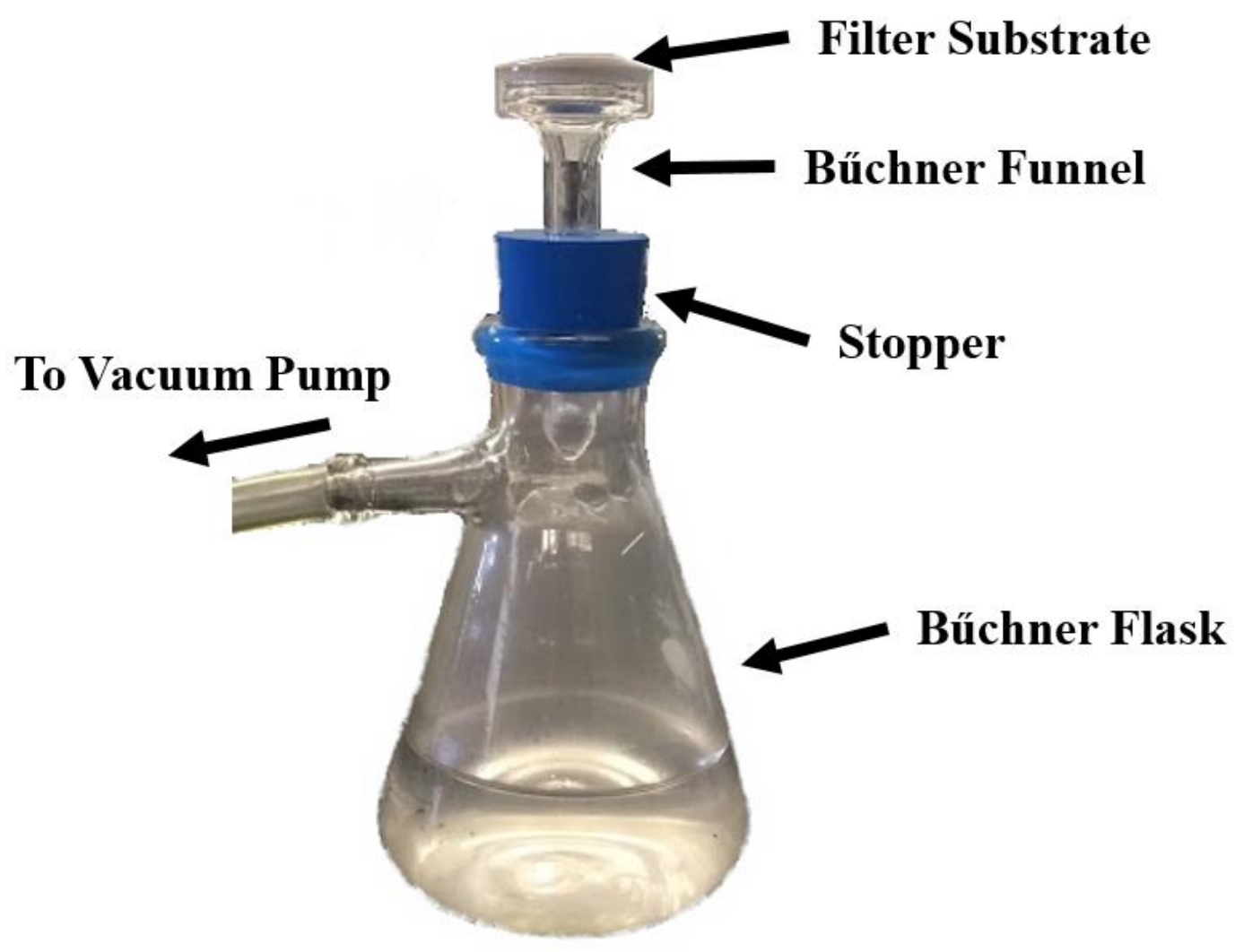

FIGURE 10. Vacuum Filtration Experimental Setup.

A Cole-Parmer Air Admiral vacuum pump was used to provide suction. One $\mathrm{mL}$ of the previously prepared $1 \mathrm{mg} / \mathrm{mL}$ graphene oxide solution was deposited on the substrate and the water was allowed to completely drain through the filter membrane. The samples were subsequently dried for 24 hours at room temperature and pressure.

\section{Flash Reduction}

The graphene oxide samples were sintered using a Xenon Corporation Double Strike linear xenon lamp. The lamp was a model LH-840 with a focus area of 2 inches by 12 inches. The lamp consisted of a xenon flash bulb backed by a parabolic focusing mirror to provide a nearly uniform radiant energy flux. The lamp was triggered with 0.58 
millisecond electrical pulses ranging in power from $150 \mathrm{~J}$ to $830 \mathrm{~J}$, as shown in FIGURE 11. The xenon lamp produced radiation in the ultraviolet and visible spectra.

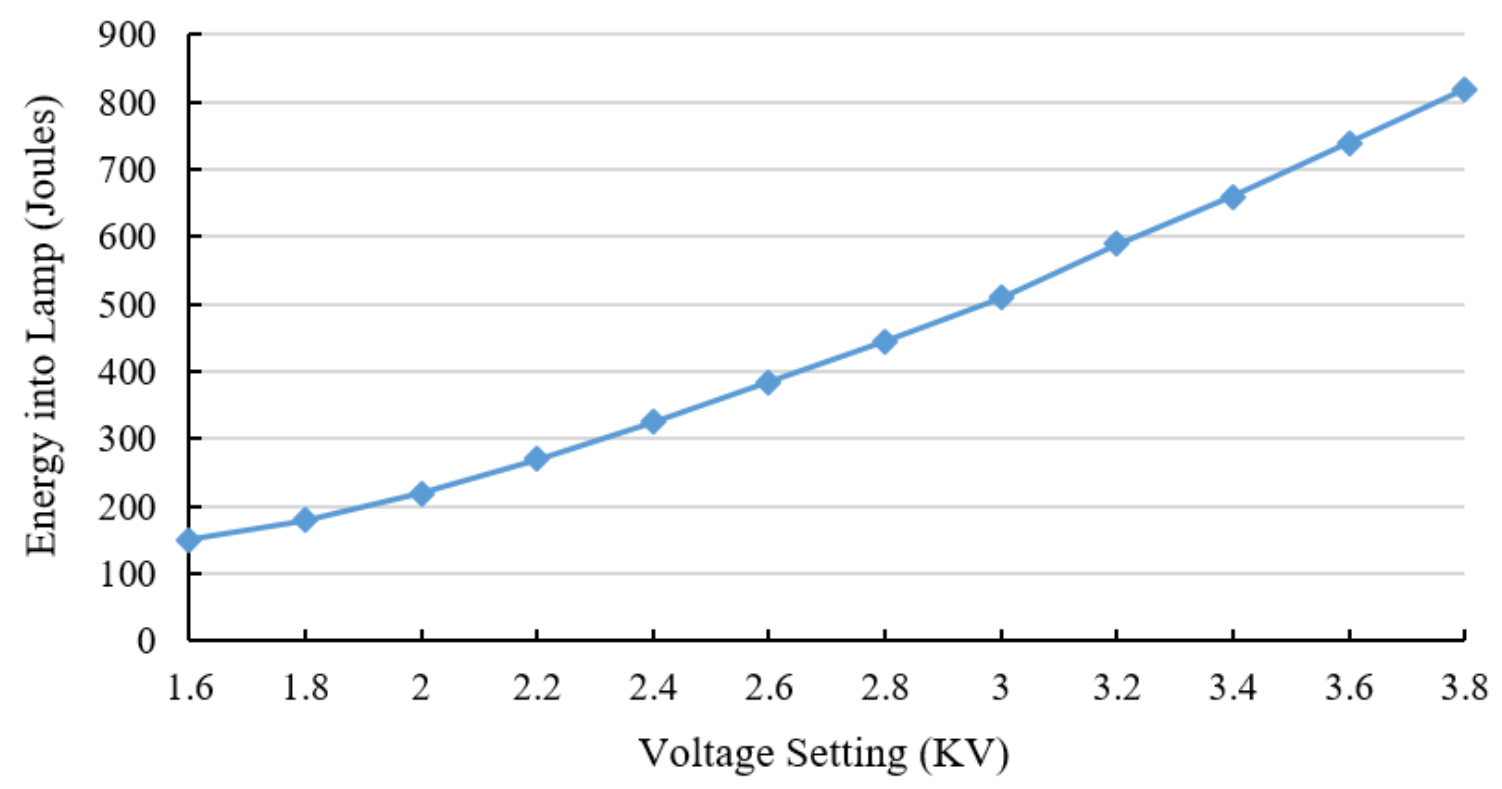

FIGURE 11. Pulse Energy at Various Power Levels for Xenon Lamp.

The samples were mounted on microscope slides to reduce curling from the substrate and for ease of transport. The samples were positioned $50 \mathrm{~mm}$ from the lamp in a specific, marked location. The radiant heat flux from the lamp varied slightly at different locations on the base plate. Positioning the samples at a marked location ensured each sample was exposed to a comparable heat flux.

\section{Filtration Testing}

The ability of graphene oxide membranes at various reduction states to reject methyl-red dye was tested. Methyl-red dye has a molecular weight of $269 \mathrm{~g} / \mathrm{mole}$ 
(NCBI 2012). This is on the size scale of sugars such as sucrose (NCBI 2018), but is an order of magnitude larger than alcohols like ethanol (NCBI 2018).

An AMI UHP-25 filtration membrane test cell was used to measure rejection of methyl-red dye by graphene oxide films. The test cell setup is shown in FIGURE 12.

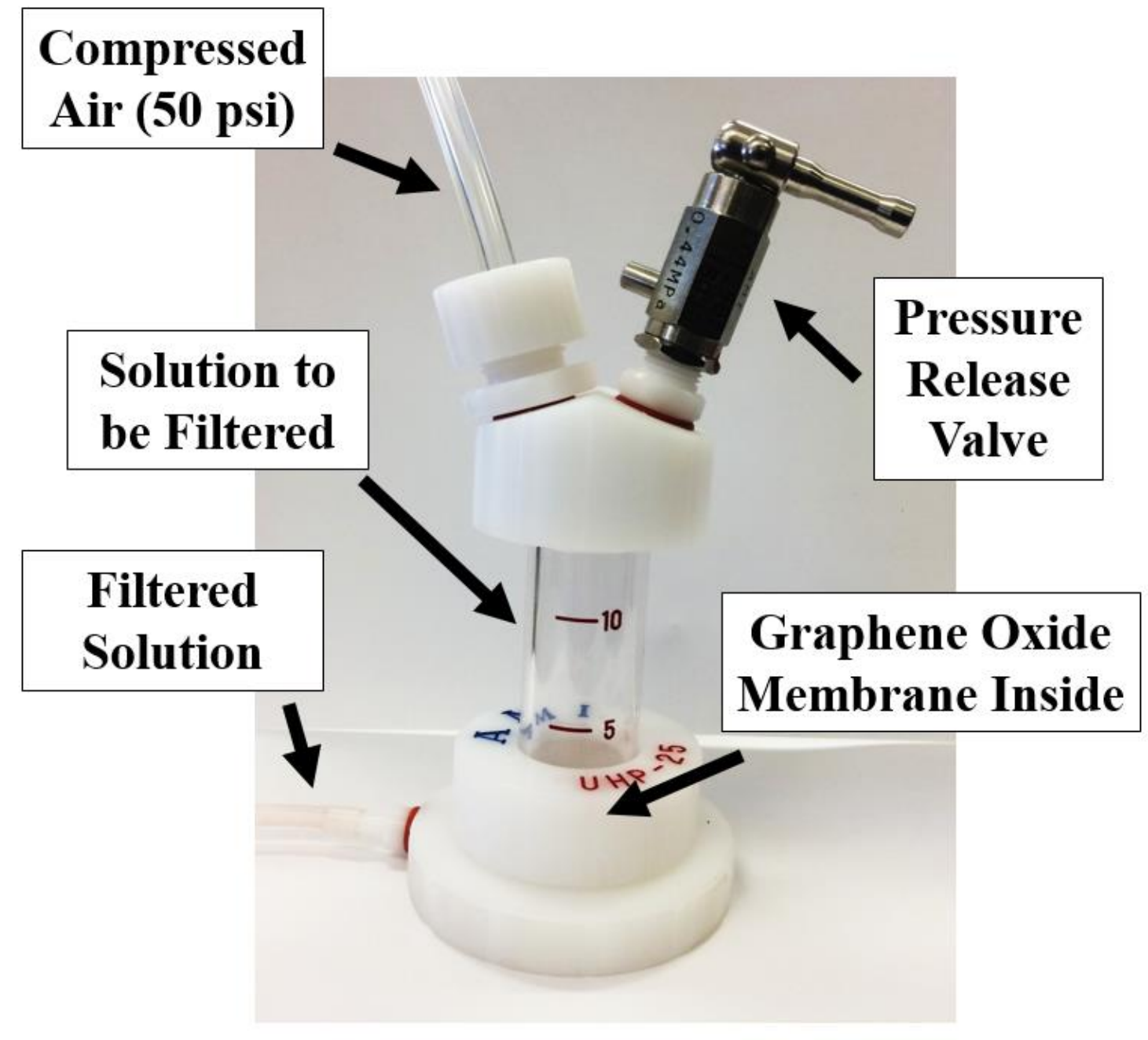

FIGURE 12. Filtration Membrane Test Cell Experiment Setup.

To make the test solution, $0.25 \mathrm{mg}$ of methyl-red dye was dispersed in $250 \mathrm{~mL}$ of DI water to form a light pink solution. $10 \mathrm{~mL}$ of this solution was placed in the test chamber for each run. Compressed air at a pressure of 50 psi was used to force the 
solution through the membrane. The membrane samples were $25 \mathrm{~mm}$ in diameter, but an O-ring used to prevent leakage reduced the area of membrane available for filtration to a $23 \mathrm{~mm}$ diameter circle. The filtered solution was collected, poured into a cuvette, and analyzed with an ultraviolet and visible light spectrophotometer to determine the change in methyl-red concentration. The test cell was rinsed twice with distilled water between filtration tests.

To determine flux through the membrane, the time required for the first $5 \mathrm{~mL}$ of solution to pass through the filter was recorded. The flux was calculated using

$$
J_{V}=\frac{\Delta V}{t \cdot A}
$$

where $J_{V}$ was the volumetric flux, $\Delta V$ was the change in solution volume in the test cell, $t$ was the time required for the first $5 \mathrm{~mL}$ of solution to pass through the filter, and $A$ was the filter area. 


\section{B. Characterization Methods}

\section{Digital Color Measurement}

The color of the samples was used as a proxy for the level of reduction of the graphene oxide. To measure color changes, the samples were photographed in a controlled lighting environment with a 5 megapixel digital camera. The lighting for the photographs was standardized by using a dark box illuminated by a single 10 lumen white LED at a set orientation to the sample. The light exposure levels for the camera were held constant for all photos.

The digital photos were analyzed with graphical software that measured the color intensity of pixels as intensity values. The pixels were measured for intensities of red, green, and blue light, on a scale from 0 to 256 . An intensity of 0 indicated no light, while an intensity of 256 indicated that the digital sensor was saturated with light. The total intensity of light was the sum of the red, green, and blue intensity values.

\section{Scanning Electron Microscopy}

The samples were imaged with a VEGA 3 Tescan and an FEI scanning electron microscope at a working depth of around $8 \mathrm{~mm}$ and $5 \mathrm{~mm}$, respectively. Samples of asdeposited graphene oxide were not sufficiently conductive for direct imaging. For these less conductive samples, a thin layer of gold was sputtered onto the surface. This gold layer increased the conductivity to the point where SEM images could be taken, but did not significantly affect the surface features of the film. 


\section{Atomic Force Microscopy (AFM)}

Surface topography for samples was measured with an Asylum Research MFP 3D atomic force microscope. The analysis was conducted in non-contact AC mode. The tip spring constant was $48 \mathrm{~N} / \mathrm{m}$ with a driving frequency of $168 \mathrm{kHz}$.

\section{Spectrophotometer Measurements}

After water with red dye was filtered with graphene oxide membranes, the resulting change in dye concentration was measured with a Perkin-Elmer Lambda 950 Ultraviolet and Visible Light Spectrophotometer. The apparatus was calibrated using a blank cuvette filled with deionized water.

The change in concentration between the unfiltered and filtered solutions was determined using Beer's Law

$$
A=\varepsilon b c
$$

where $A$ was the absorbance of the solution, $\varepsilon$ was the molar absorptivity of the solute, $b$ was the path length through the sample, and $c$ was the concentration of the solute.

The same solute was dissolved in all tested samples, so $\varepsilon$, the molar absorptivity

of the solute, was the same for all samples. The intensity spectra from the spectrophotometer tests were all maximized at the same spectra, further indicating that all the samples had similar molar absorptivity characteristics. Additionally, $b$, the path length, was the same for all samples because the same cuvette size was used for all tests. 
Because $\varepsilon$ and $b$ were common for all samples, the relative concentrations between samples could be expressed as

$$
\frac{A_{1}}{A_{2}}=\frac{c_{1}}{c_{2}}
$$

where $A_{1}$ and $A_{2}$ are the absorptivity of two samples, and $c_{1}$ and $c_{2}$ are the concentrations of the two samples. Equation 4 allowed relative concentrations of dye to be determined based on the height of the absorbance curve measured by the spectrophotometer. 


\section{RESULTS AND DISCUSSION}

\section{A. Digital Color Measurement}

As seen in FIGURE 13, the as-deposited graphene oxide film was a semitransparent light brown, only absorbing a small amount of red, blue, and green light. As the film was reduced, it visibly darkened and turned a darker brown. The change in color was a proxy for the reduction state of the graphene oxide film.

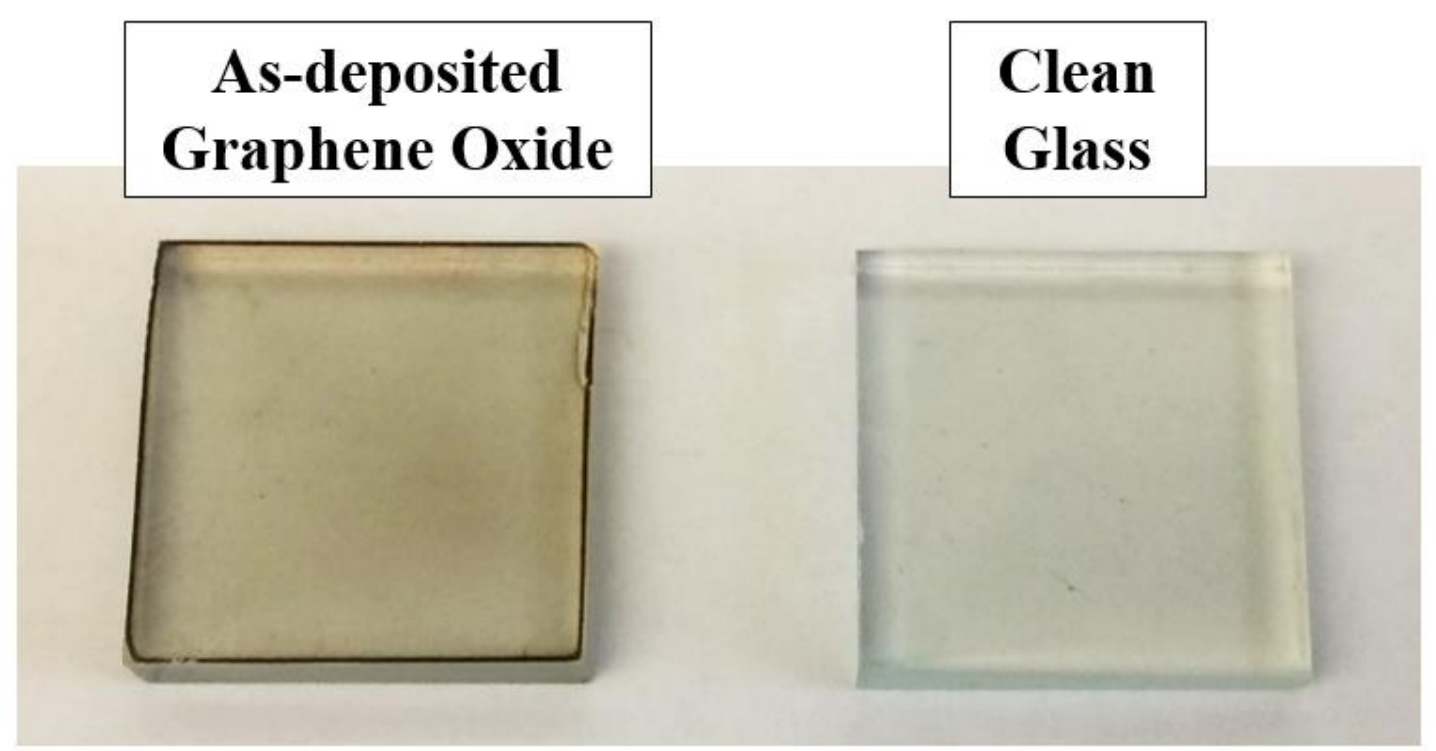

FIGURE 13. As-Deposited Graphene Oxide on Glass.

In preliminary tests, three regions of color change were observed. As displayed in FIGURE 14, at the lowest power setting no significant change in color was observed. In the range from $1.8 \mathrm{kV}$ to $2.2 \mathrm{kV}$, the film darkened uniformly. At power levels of $2.4 \mathrm{kV}$ and above, dark speckles of color appeared. 


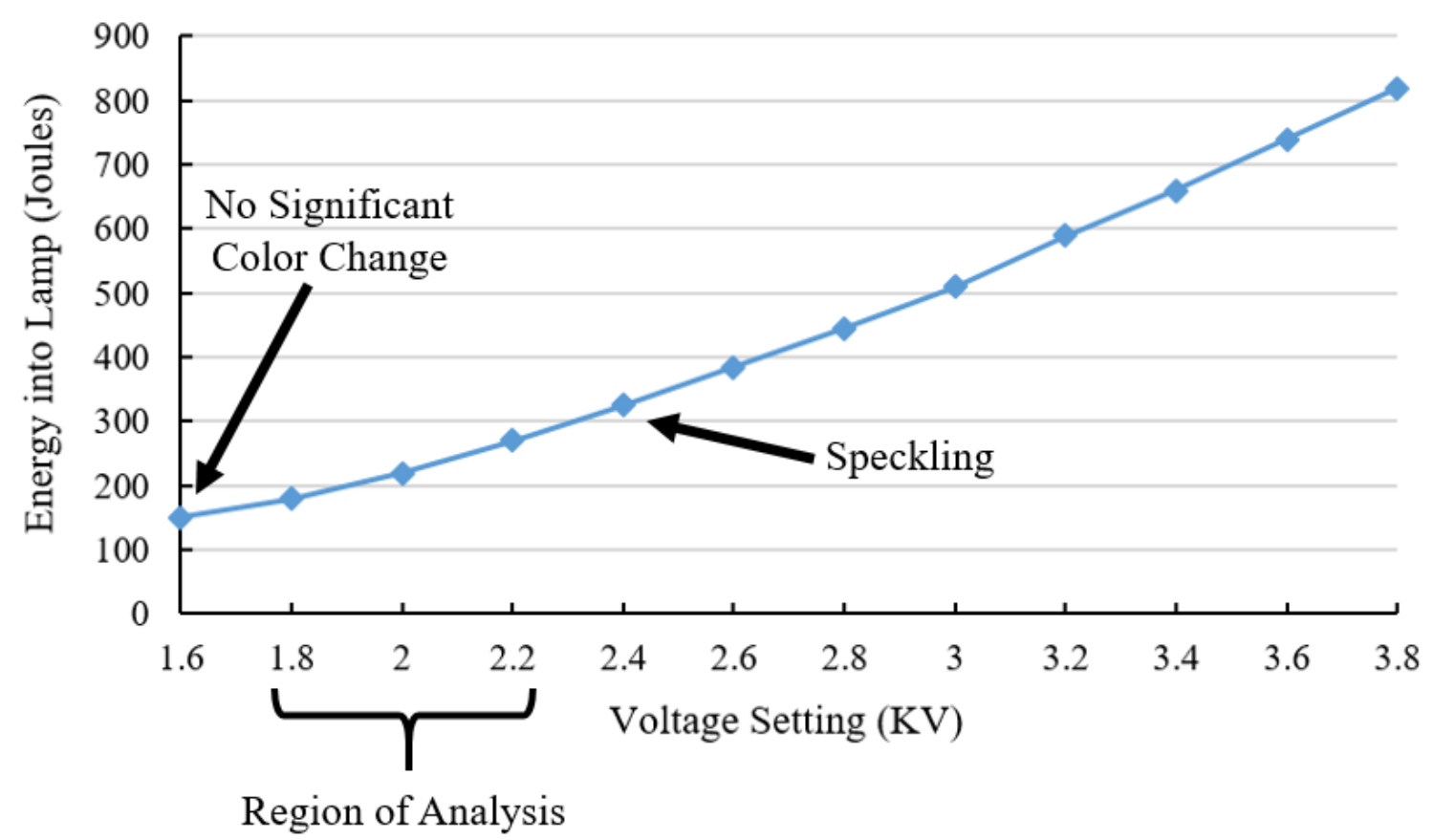

FIGURE 14. Xenon Lamp Power Setting Region for Color Analysis.

Because the speckled areas displayed a dramatic increase in electrical conductivity, they appeared to be areas with high levels of reduction (Pei 2012).

However, samples with these speckles displayed poor filtration performance. Because this study focused on the filtration properties of graphene oxide films, pulses at power levels high enough to cause speckles were not used for the color change study.

In order to measure the influence of reduction level on graphene oxide film color, 36 graphene oxide film samples were prepared, as seen in FIGURE 15. 


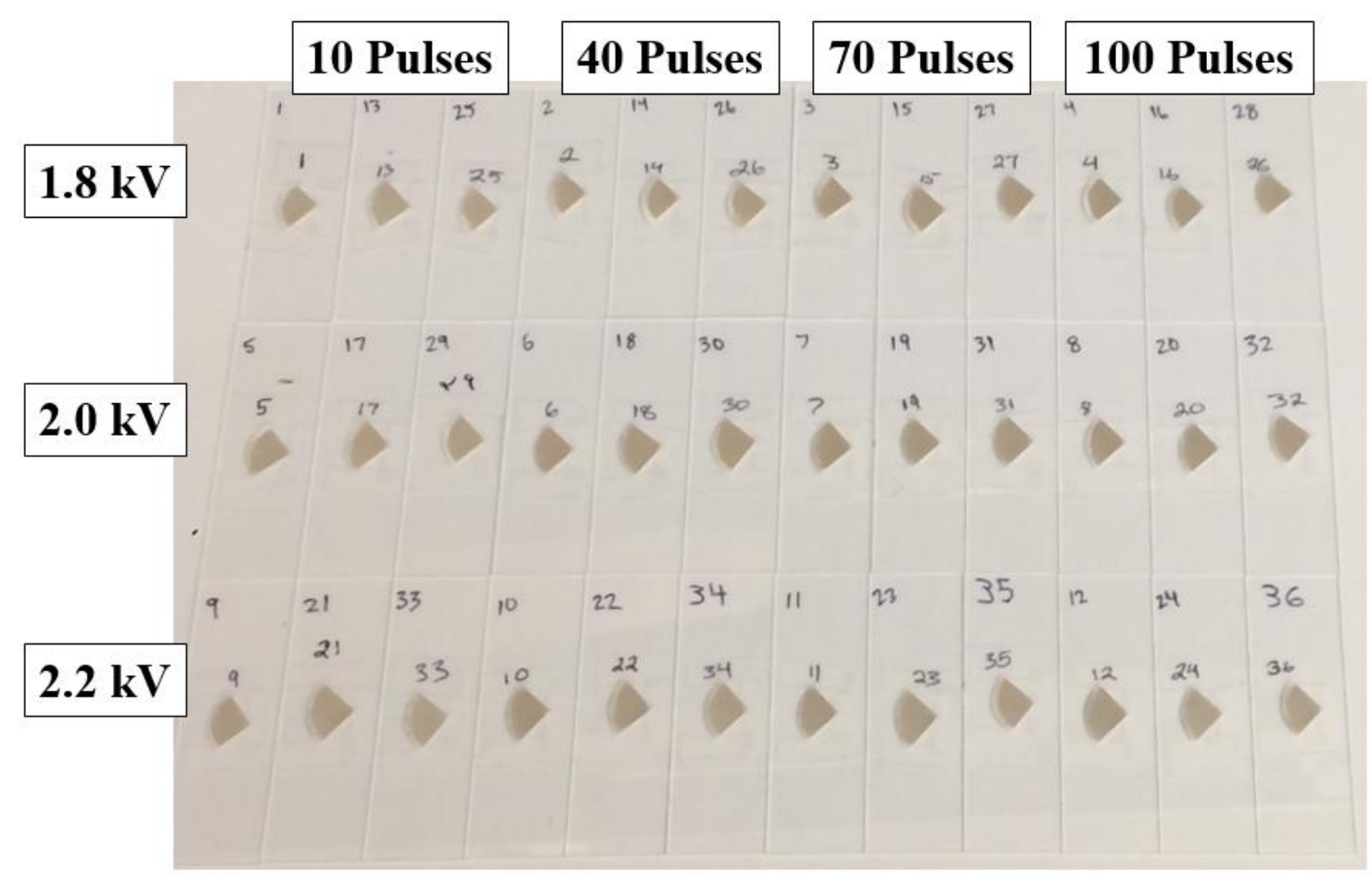

FIGURE 15. Graphene Oxide Films Exposed to Various Pulse Powers and Amounts.

The prepared samples were exposed to $10,40,70$, and 100 pulses at power levels of $1.8 \mathrm{kV}, 2.0 \mathrm{kV}$, and $2.2 \mathrm{kV}$. After reduction, the graphene oxide samples were photographed in a controlled lighting environment. The images of the samples were analyzed using photo editing software that listed the RGB color levels for pixels in standard regions of the samples. The raw data is listed in TABLE III in Appendix I.

As the graphene oxide reduced, it absorbed more light in higher wavelengths, especially blue and ultraviolet (Pei 2012). Since more of the blue light was absorbed, less was reflected to the camera, resulting in a decrease in measured blue intensity. The changes in blue light intensity for samples exposed to various power levels and pulse numbers are listed in FIGURE 16. 


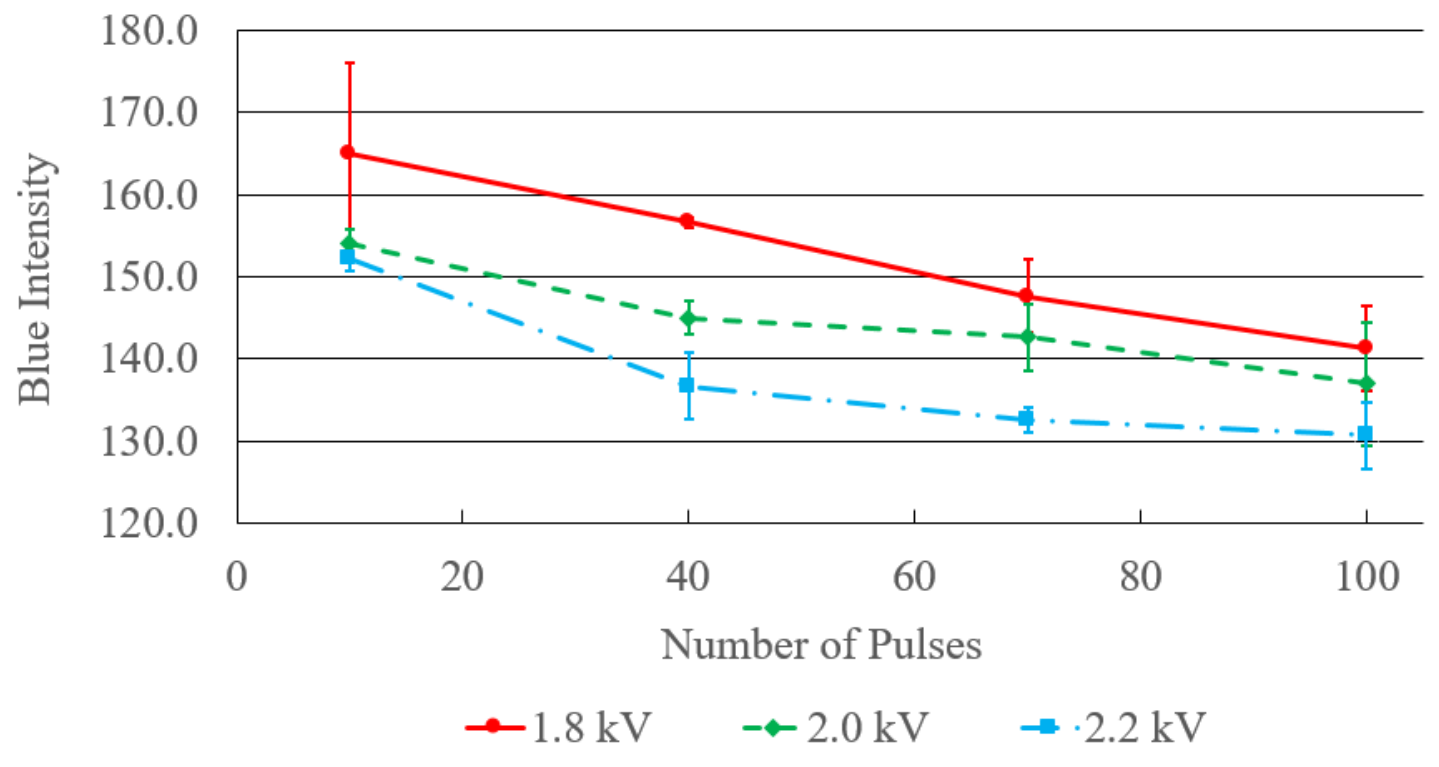

FIGURE 16. Blue Light Intensity at Various Pulse and Power Levels.

The blue color intensity for samples across all three power setting decreased as the samples were reduced and darkened in color. The higher temperatures caused by higher power levels of the xenon lamp caused the graphene oxide to darken more quickly. At 70 and 100 pulses there is a region of reduced change in sample color. This appears to be the result of the sample reaching a temperature plateau similar to those predicted in simulations of intense pulse light treatments (Druffel, Dharmadasa et al. 2018). 


\section{B. $\underline{\text { SEM Images }}$}

SEM images were used to determine the cross-sectional thickness of the asdeposited graphene oxide film. The entire film cross-section is shown in FIGURE 17.

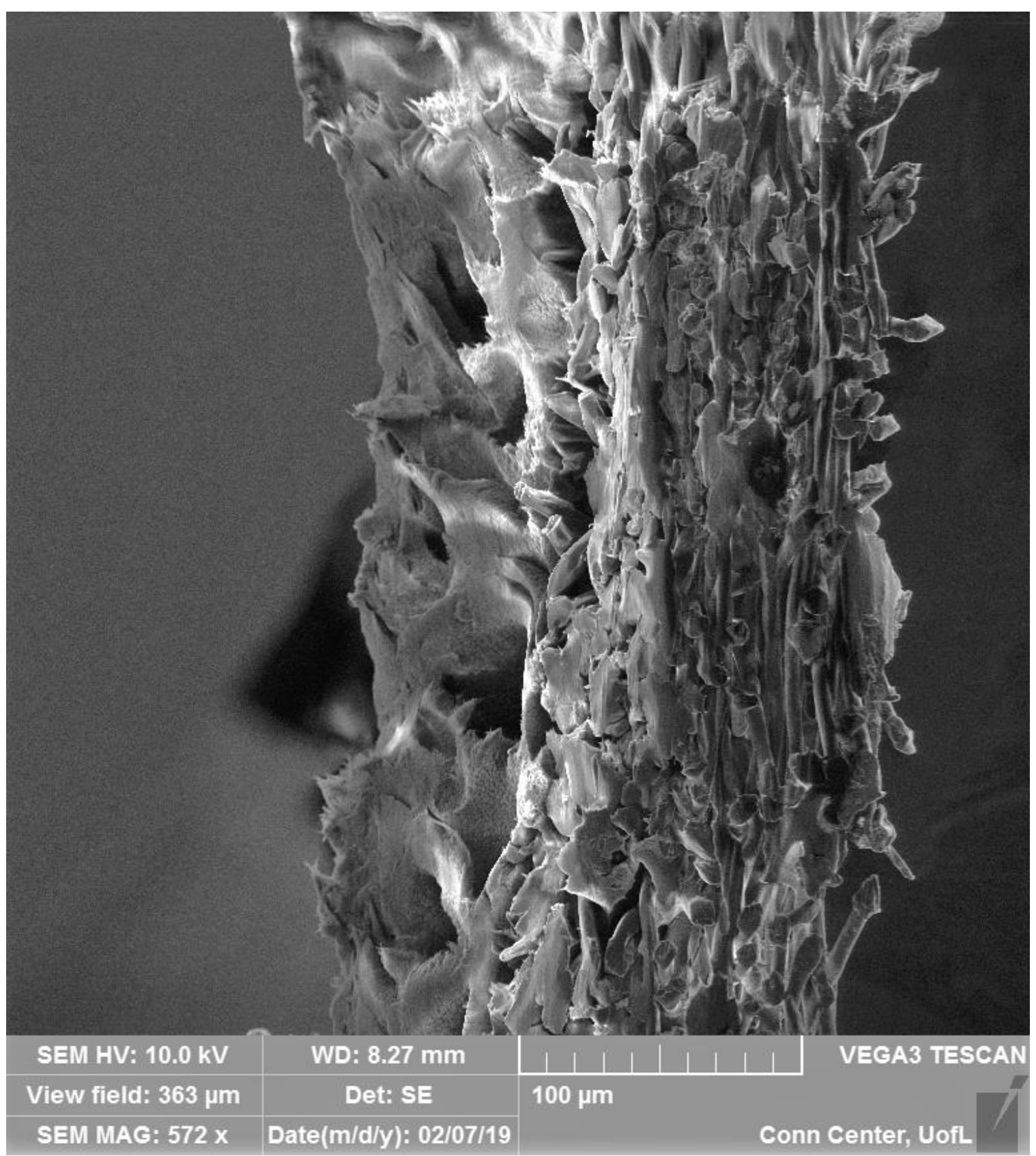

FIGURE 17. Cross Section of Graphene Oxide Film and PVDF Filter Paper. 
The darker fibers on the right in FIGURE 17 are the cellulose backing of the substrate. The lighter fibers near the center of the image make up the polymer coating that supports the graphene oxide film. The actual graphene oxide cross-section is too small to be visible from this view.

A detail view of the graphene oxide film is shown in FIGURE 18. Note that the sample is oriented horizontally in FIGURE 18, instead of vertically, as in FIGURE 17.

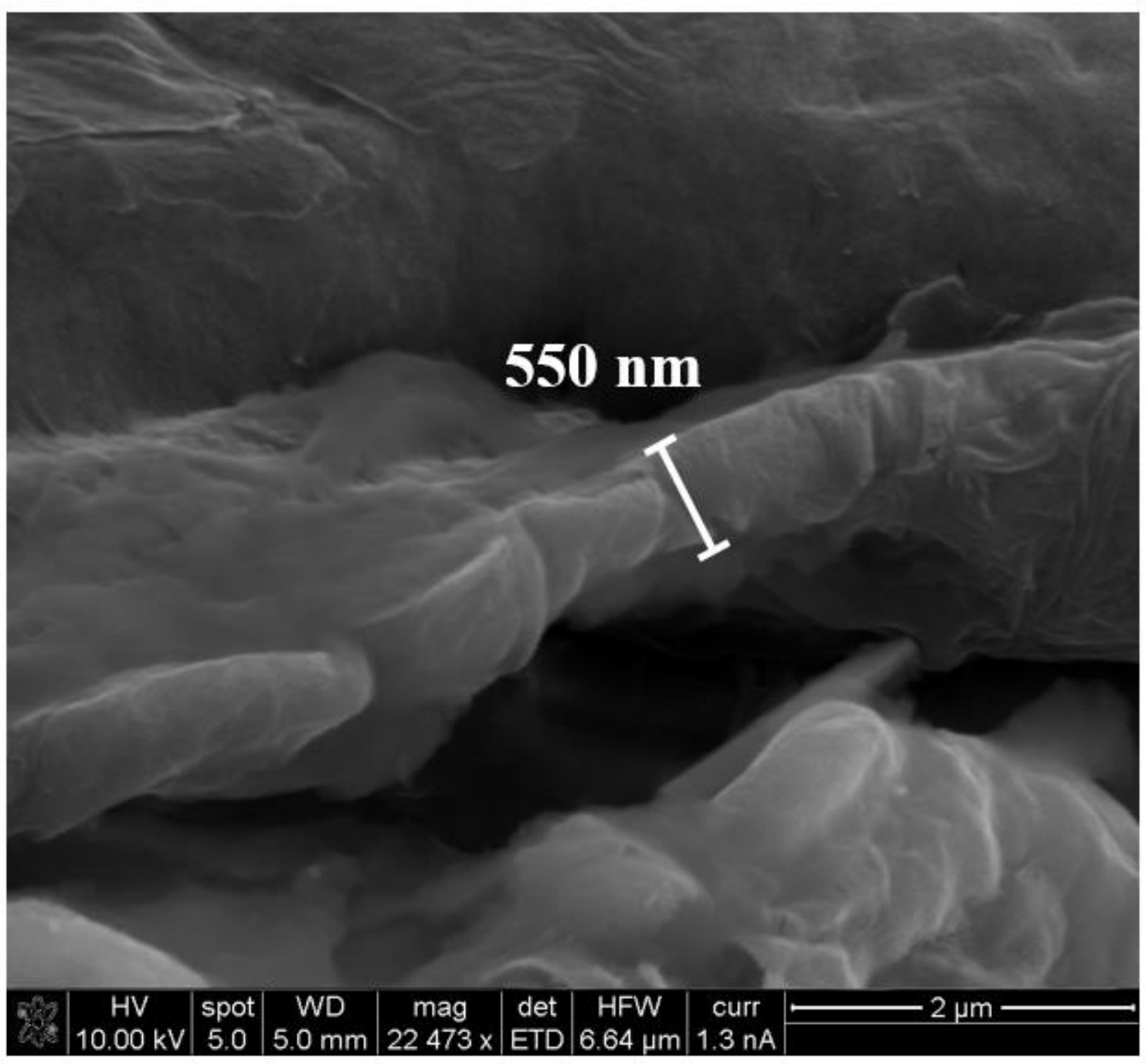

FIGURE 18. Cross Section SEM Image of As-Deposited Graphene Oxide Film. 
In FIGURE 18, the graphene oxide film has been separated from the substrate during the cutting and preparation of the cross-section sample. Pixel measurements made with graphical analysis software at several points in multiple images of the film determined the average cross-sectional thickness to be $550 \mathrm{~nm}$. 


\section{AFM Topography}

Samples of as-deposited graphene oxide, reduced graphene oxide, and plain polymer were analyzed with AFM. The analysis was conducted in non-contact AC mode with a tip spring constant of $48 \mathrm{~N} / \mathrm{m}$. The samples were analyzed over 12 micron square and 2 micron square areas. The fast scan direction for all images was horizontal, and the slow scan direction was vertical. The samples were rotated $45^{\circ}$ with no significant change in the measured surface topography. The following images are amplitude scans, as this mode revealed the most surface detail.

The first three scans were conducted over a 12 micron square area. The first scan shows the untreated polymer substrate in FIGURE 19. 


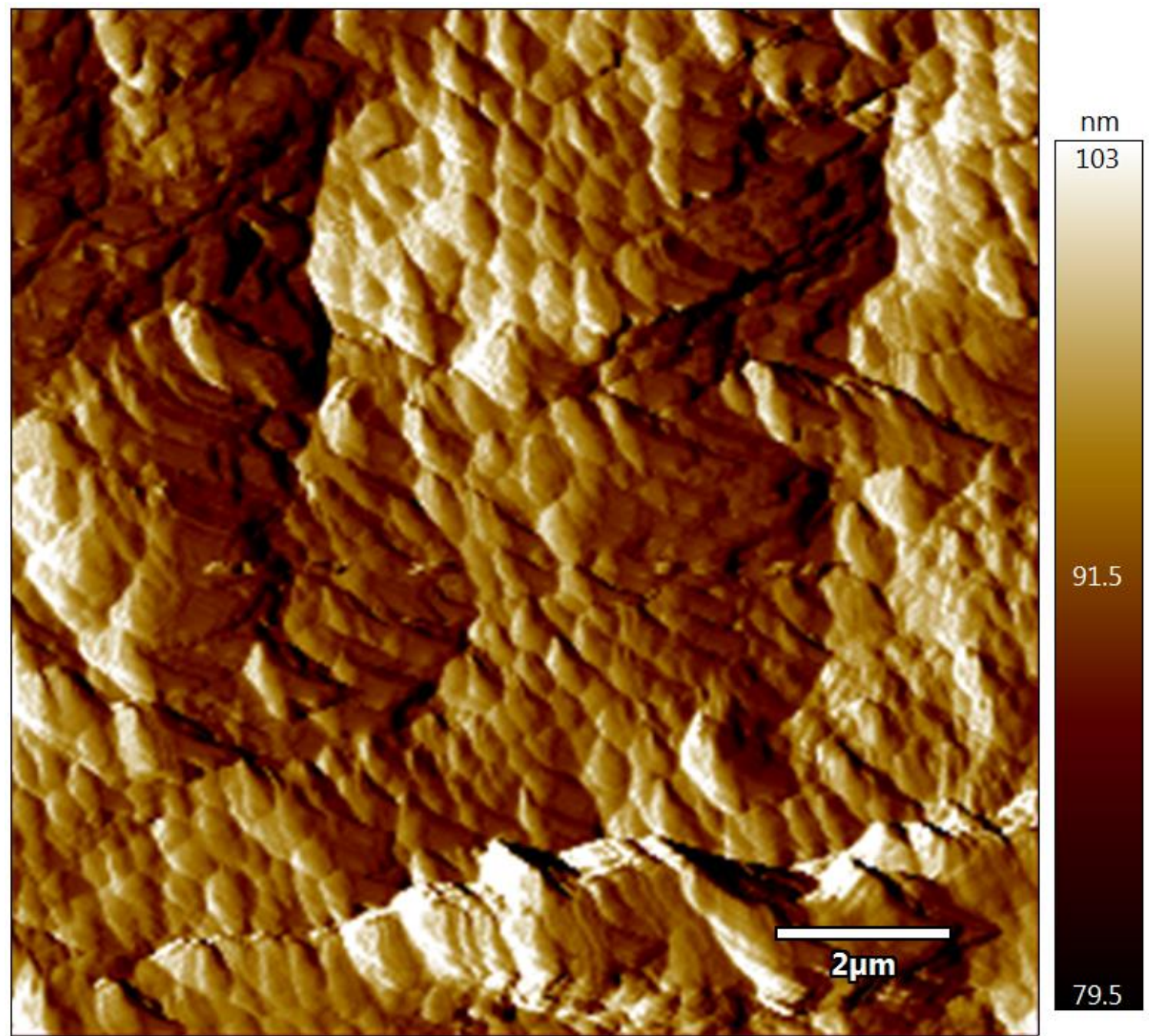

FIGURE 19. Twelve Micron Square Scan of Untreated Polymer Substrate.

At this magnification, the untreated polymer substrate consists of $500 \mathrm{~nm}$ scalelike features that are distributed over larger ridges and valleys in the bulk polymer layer. A more focused scan of the untreated polymer substrate in FIGURE 20 shows a two micron square section. 


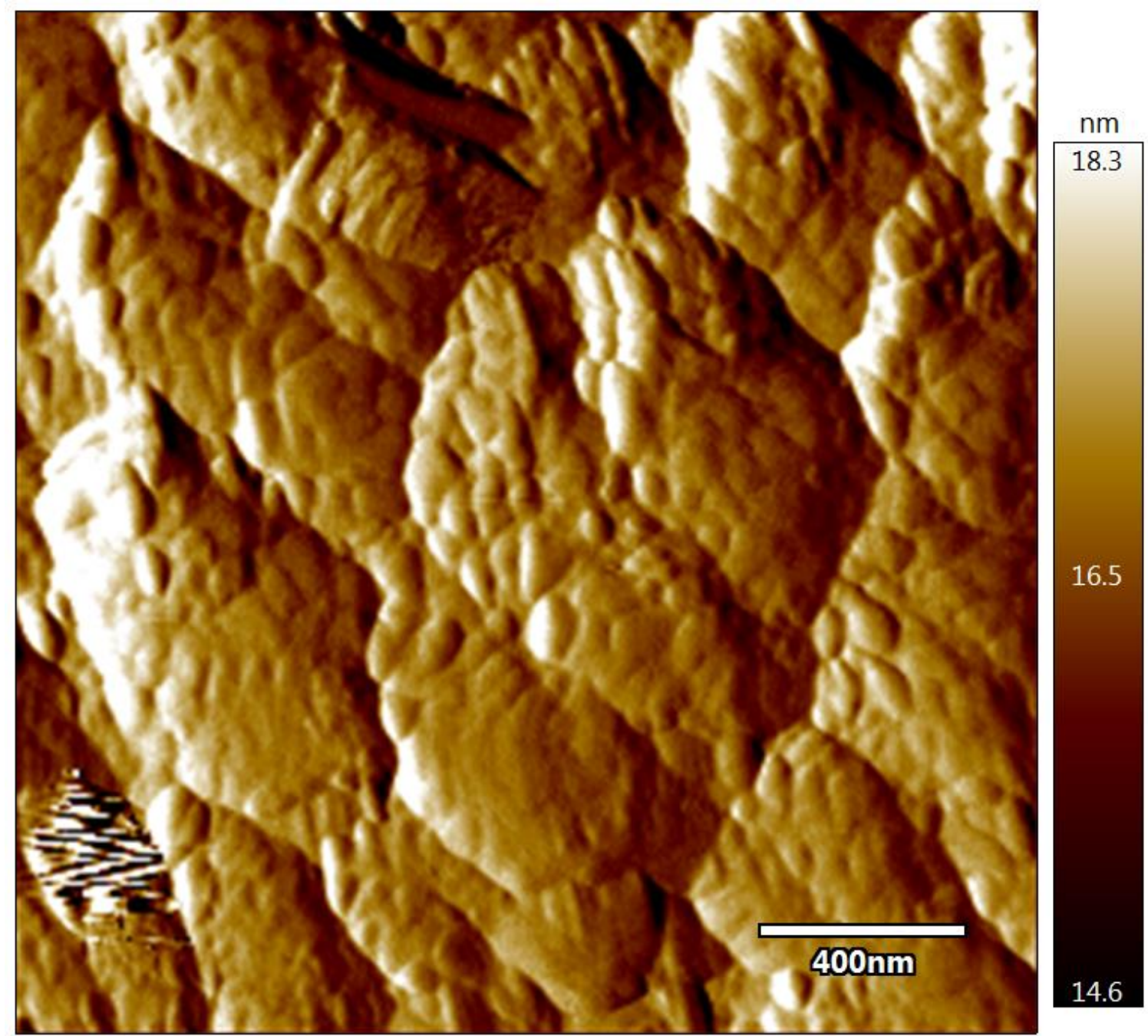

FIGURE 20. Two Micron Square Scan of Untreated Polymer Substrate.

At this magnification, the individual scale-like features visible in FIGURE 19 are displayed in more detail. The scale-like features are marked by 30-50 nm lumps of polymer that were joined together when the polymer cured.

After graphene oxide was deposited on the surface of the polymer substrate, a change in surface topography was observed. FIGURE 21 shows the first scan of the graphene oxide film. 


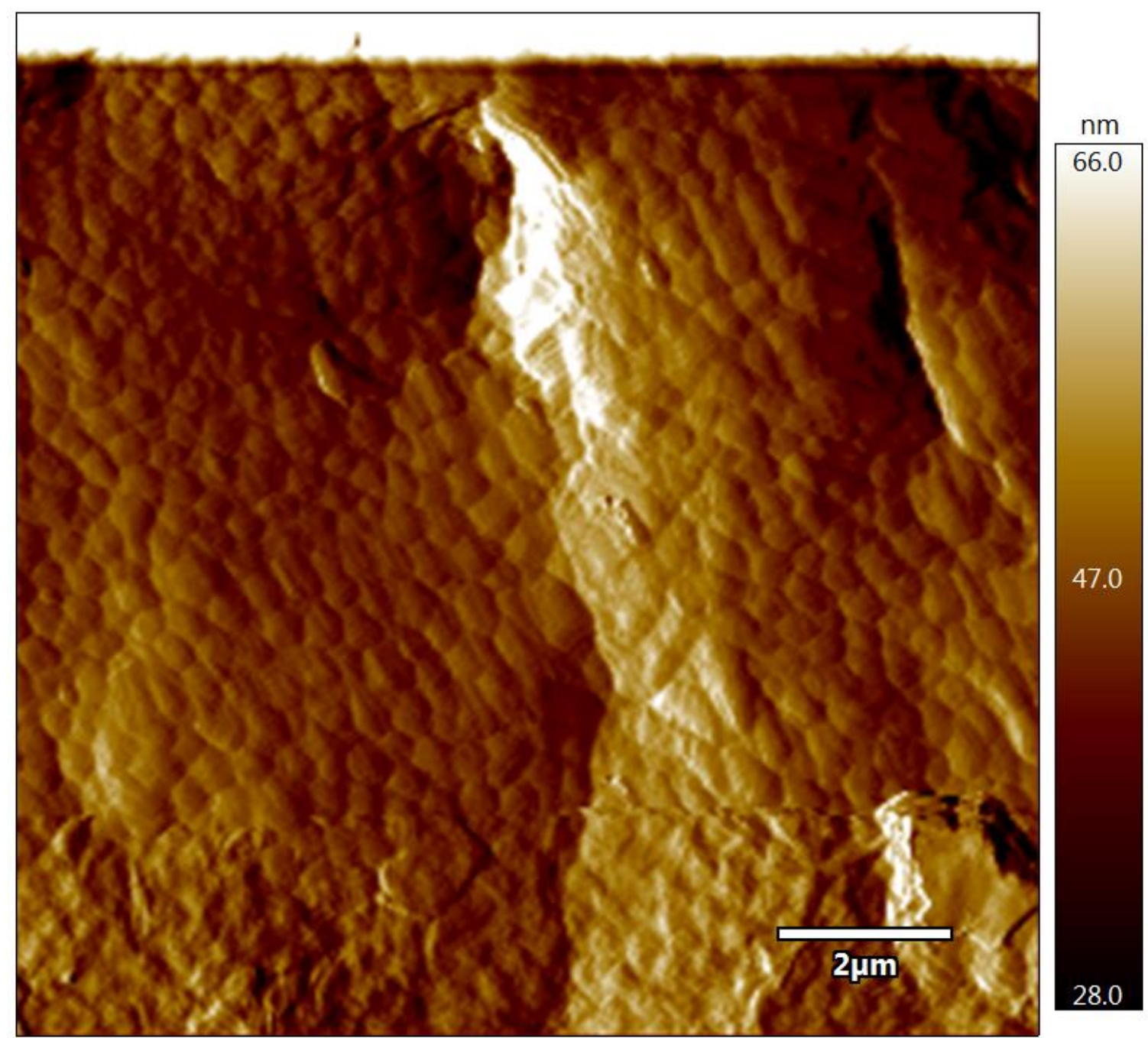

FIGURE 21. Twelve Micron Square Scan of As-Deposited Graphene Oxide Film.

At this magnification, the scale-like features visible in FIGURE 19 are still present, although they are less well defined. The deposited graphene oxide film conformed to the major surface features of the polymer substrate, but it obscured the deeper valleys between the polymer lumps.

The change in surface topography is more evident at higher magnification. FIGURE 22 shows individual flakes of graphene oxide deposited on the substrate. 


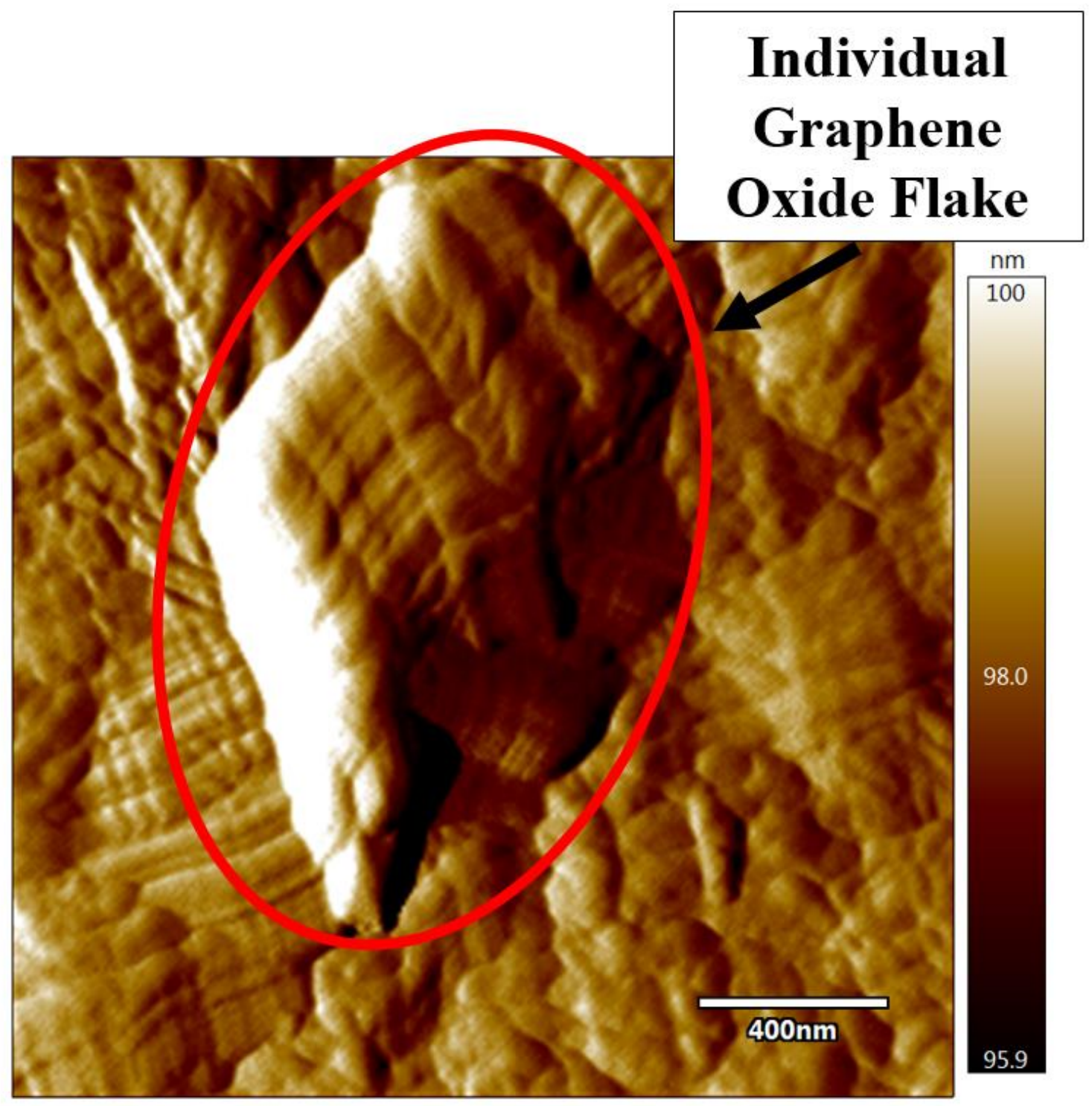

FIGURE 22. Two Micron Square Scan of As-Deposited Graphene Oxide.

Instead of the bumpy polymer surface in FIGURE 20, the striated surfaces of graphene oxide flakes are visible. An individual flake is highlighted, and is consistent with the size of flake listed in the data sheet from the graphene oxide supplier (Graphenea 2017).

A 12 micron square scan of a reduced graphene oxide film area is shown in FIGURE 23. This sample pictured was reduced at high power to achieve a high level of 
reduction. It should be noted that the samples used in the filtration study were more lightly reduced and had a topography similar to FIGURE 21.
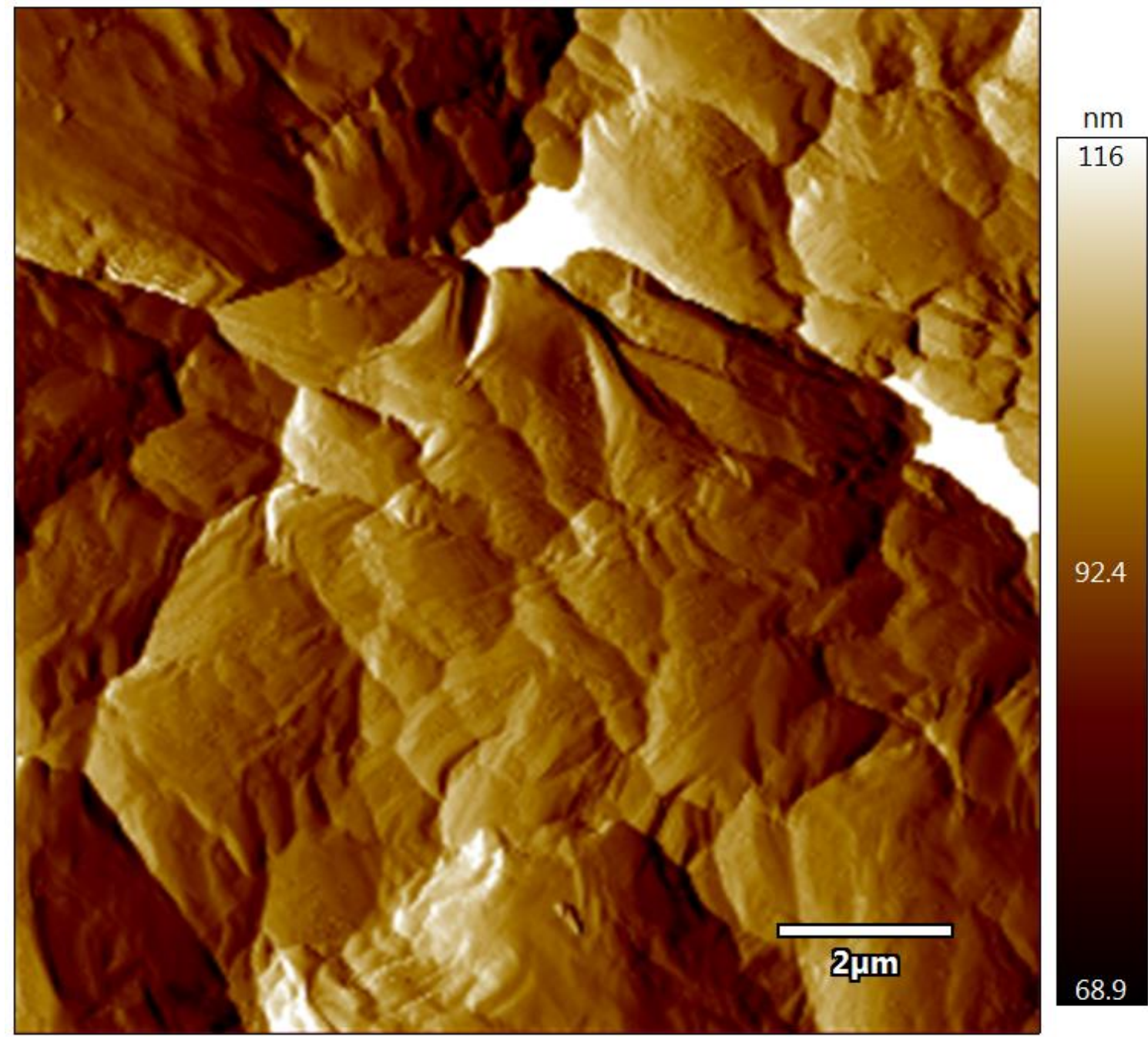

FIGURE 23. Twelve Micron Square Scan of Reduced Graphene Oxide Film.

The reduced graphene oxide film no longer conformed to the general topography of the polymer substrate. The graphene oxide flakes have been sintered together, but they also show significant wrinkles in their surface as a result of the reduction reaction.

The magnified view of the reduced graphene oxide film is shown in FIGURE 24. 


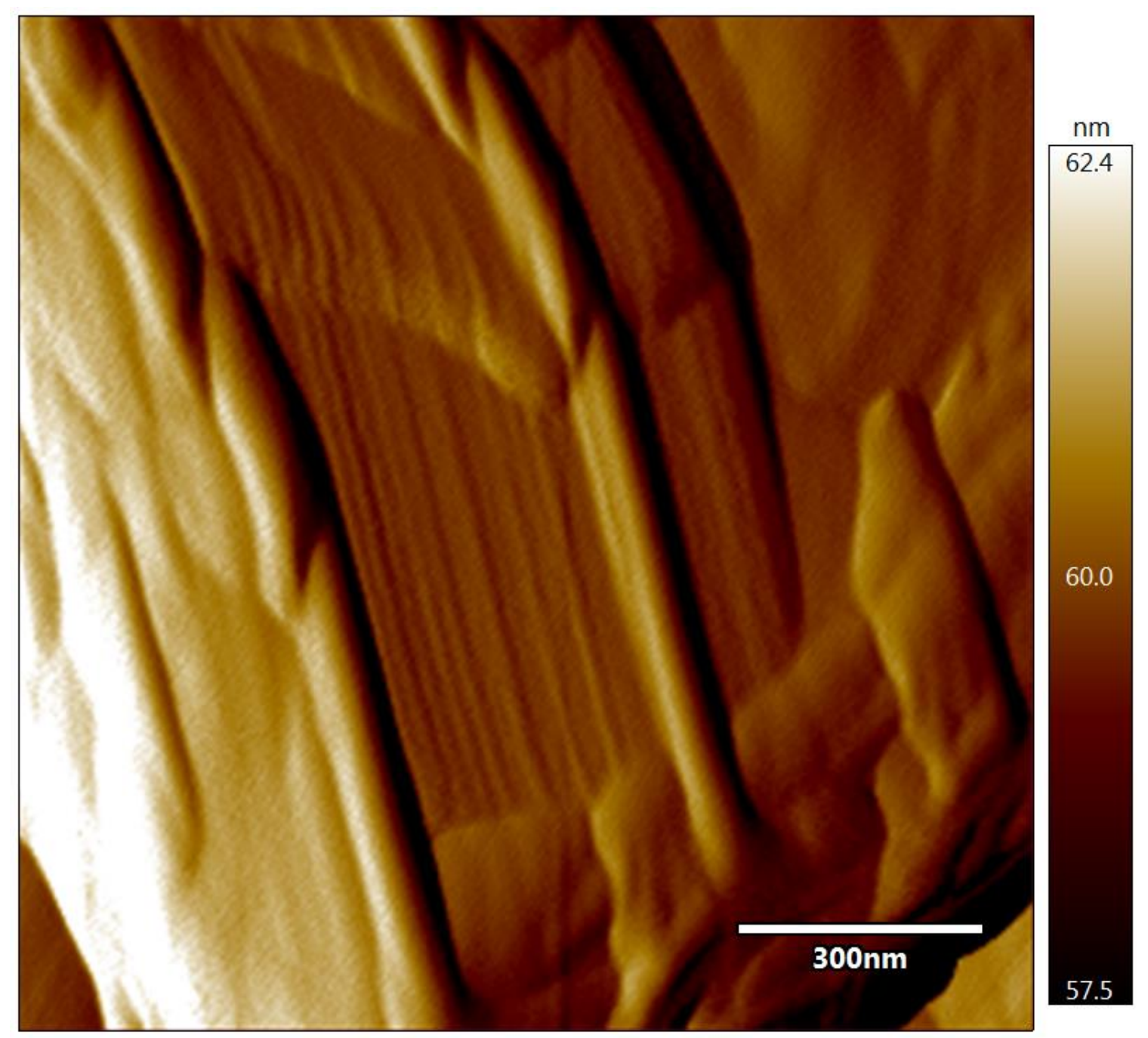

FIGURE 24. Two Micron Scan of Reduced Graphene Oxide Film.

At greater magnification, the surface wrinkles in the graphene oxide film are clearer, as well as some regular striations in the center of the image. The spacing of the striations appears to be around $15-30 \mathrm{~nm}$, which is consistent with the estimated thickness of the graphene oxide sheets. The total width of the cross section appears to be around $500 \mathrm{~nm}$, which is consistent with the SEM cross-section images. In this image, it appears the graphene oxide film has buckled, exposing a cross-section of the molecular sheets. 


\section{Spectrophotometer Measurement}

A membrane test cell was used to test the filtration performance of graphene oxide membranes at various stages of reduction. Graphene oxide films were prepared by exposing them to xenon lamp pulses at a power setting of $2.2 \mathrm{kV}$. Samples were exposed to 5, 10, and 15 pulses resulting in the films shown in FIGURE 25.
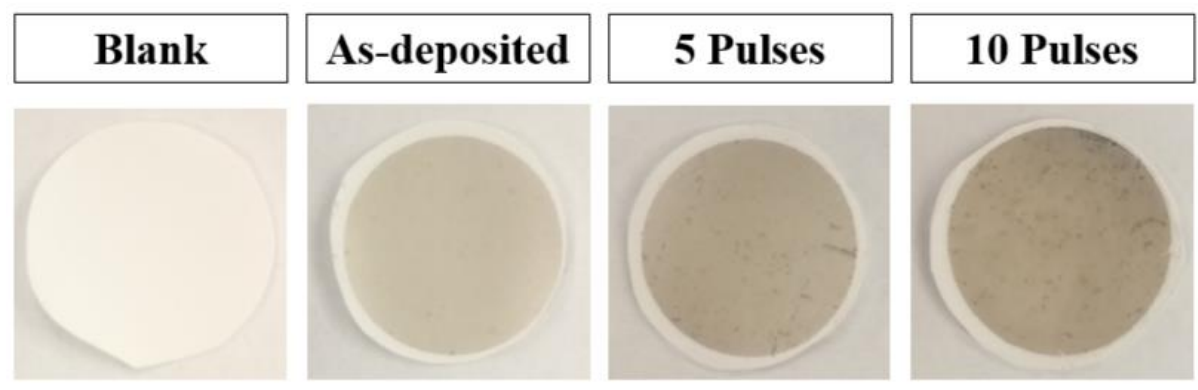

\section{Pulses}

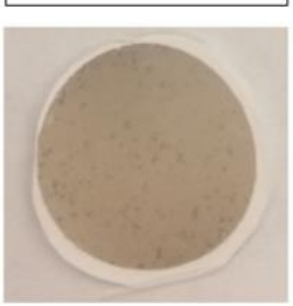

FIGURE 25. Graphene Oxide Samples Prepared for Filtration Testing.

The flux through the filter material was measured by recording how long the first $5 \mathrm{~mL}$ of solution took to pass through the filter. The reliability of the flow times was limited by the precision of the volume measurement on the test cell, which was $\pm 0.2 \mathrm{~mL}$. Because of the precision limitation from the volume reading, the flow times are reliable $\pm 4 \%$. The time and flux for each filter material is displayed in TABLE I. 
TABLE I

FLOW RATE AND FLUX FOR FILTER SAMPLES.

\begin{tabular}{|l|c|c|c|}
\hline \multicolumn{1}{|c|}{ Sample Type } & Number of Pulses & Flow Time (min:s) & Flux LMH $\left(\frac{L}{m^{2} \cdot h r}\right)$ \\
\hline Blank Substrate & - & $00: 10$ & 4320 \\
\hline Graphene Oxide & 0 & $60: 00$ & 12.0 \\
\hline Graphene Oxide & 5 & $08: 55$ & 80.7 \\
\hline Graphene Oxide & 10 & $04: 45$ & 152 \\
\hline Graphene Oxide & 20 & $00: 05$ & 8640 \\
\hline
\end{tabular}

The flux for each filter material was calculated using Equation 2. The asdeposited graphene oxide had the fewest defects and smallest pore size, making its flux the smallest. The light pulses removed oxygen groups and introduced defects into the graphene oxide film, so films that were exposed to more light pulses had higher flux.

The color of the filtered solutions was measured with a spectrophotometer, and the results are displayed in FIGURE 26. 


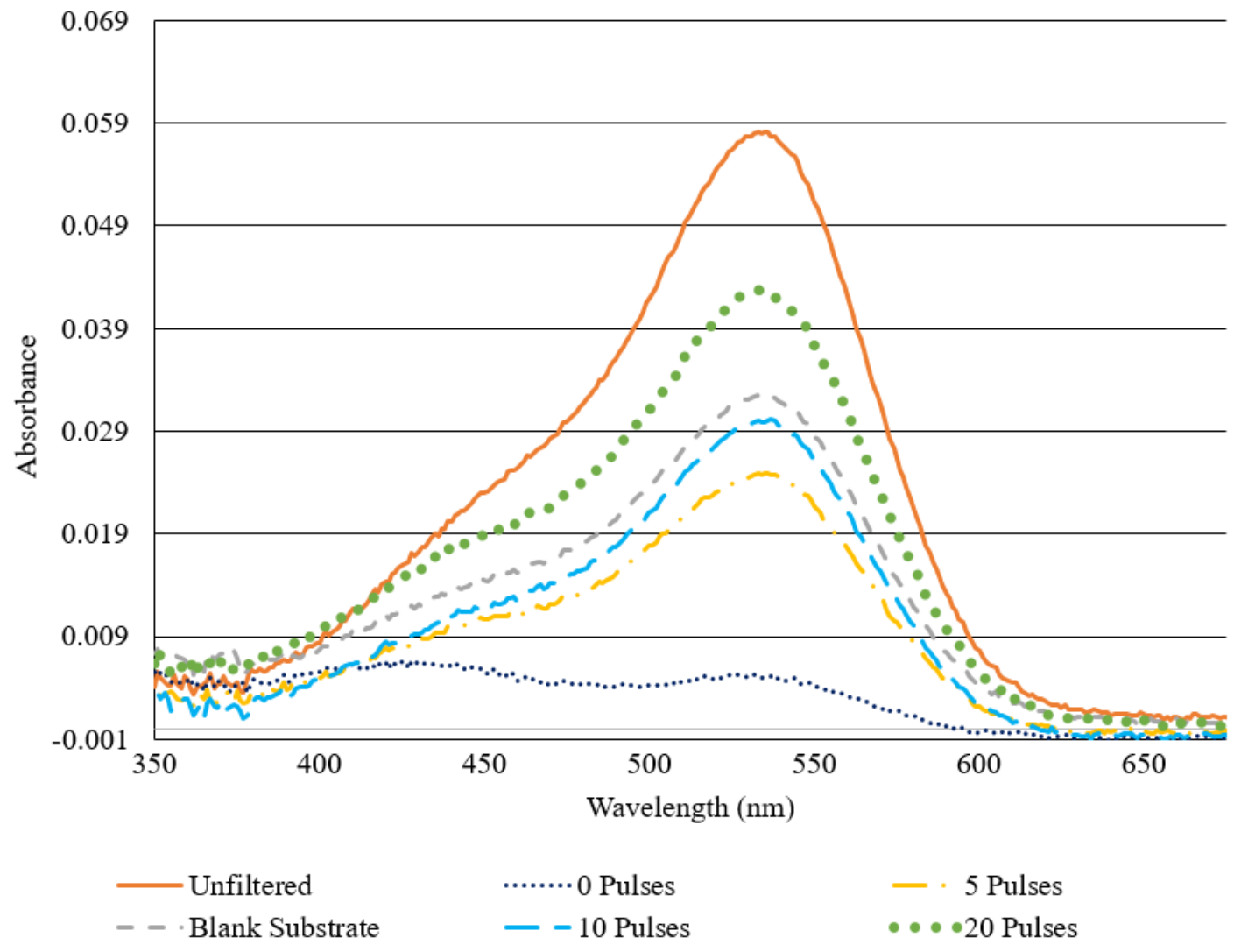

FIGURE 26. Filtered Solution Absorbance for Visible and Ultraviolet Spectra.

The methyl-red dye caused the absorbance peak at $535 \mathrm{~nm}$ in FIGURE 26, and the heights of the absorbance peaks at $535 \mathrm{~nm}$ were linearly proportional to the concentration of the dye. The change in the height of the absorbance peak after filtration indicated the amount of filtrate rejected by the membrane. The percentage rejection was calculated with

$$
\% R=\frac{A_{\text {unfiltered }}-A_{i}}{A_{\text {unfiltered }}}
$$

where $\% R$ was the percent rejection, $A_{\text {unfiltered }}$ was the height of the absorbance peak of the unfiltered solution, and $A_{i}$ was the absorbance peak of the filtered solution. The 
heights of the absorbance peaks and percent dye rejection values are displayed in TABLE

II.

TABLE II.

PEAK ABSORBANCE AND DYE REJECTION FOR FILTERED SOLUTIONS.

\begin{tabular}{|c|c|c|}
\hline Sample Type & Peak Absorbance & Dye Rejection (\%) \\
\hline Unfiltered & 0.0582 & - \\
\hline Blank Substrate & 0.0325 & $44.1 \%$ \\
\hline As-deposited & 0.0053 & $90.9 \%$ \\
\hline 5 Pulses & 0.0249 & $57.2 \%$ \\
\hline 10 Pulses & 0.0301 & $48.2 \%$ \\
\hline 20 Pulses & 0.0427 & $26.5 \%$ \\
\hline
\end{tabular}

The as-deposited graphene oxide rejected the highest percentage of red dye because of the low defect rate in the membrane. The light pulses introduced more defects into the graphene oxide film, causing less dye to be rejected. Interestingly, the rejection percentage for the samples exposed to 20 light pulses is lower than the blank substrate, indicating that the light pulses may have damaged the substrate either by melting or oxidation.

The overall filtration performance of a reduced graphene oxide membrane is a combination of flux and dye rejection percentage. These properties are compared for each sample tested in FIGURE 27. 


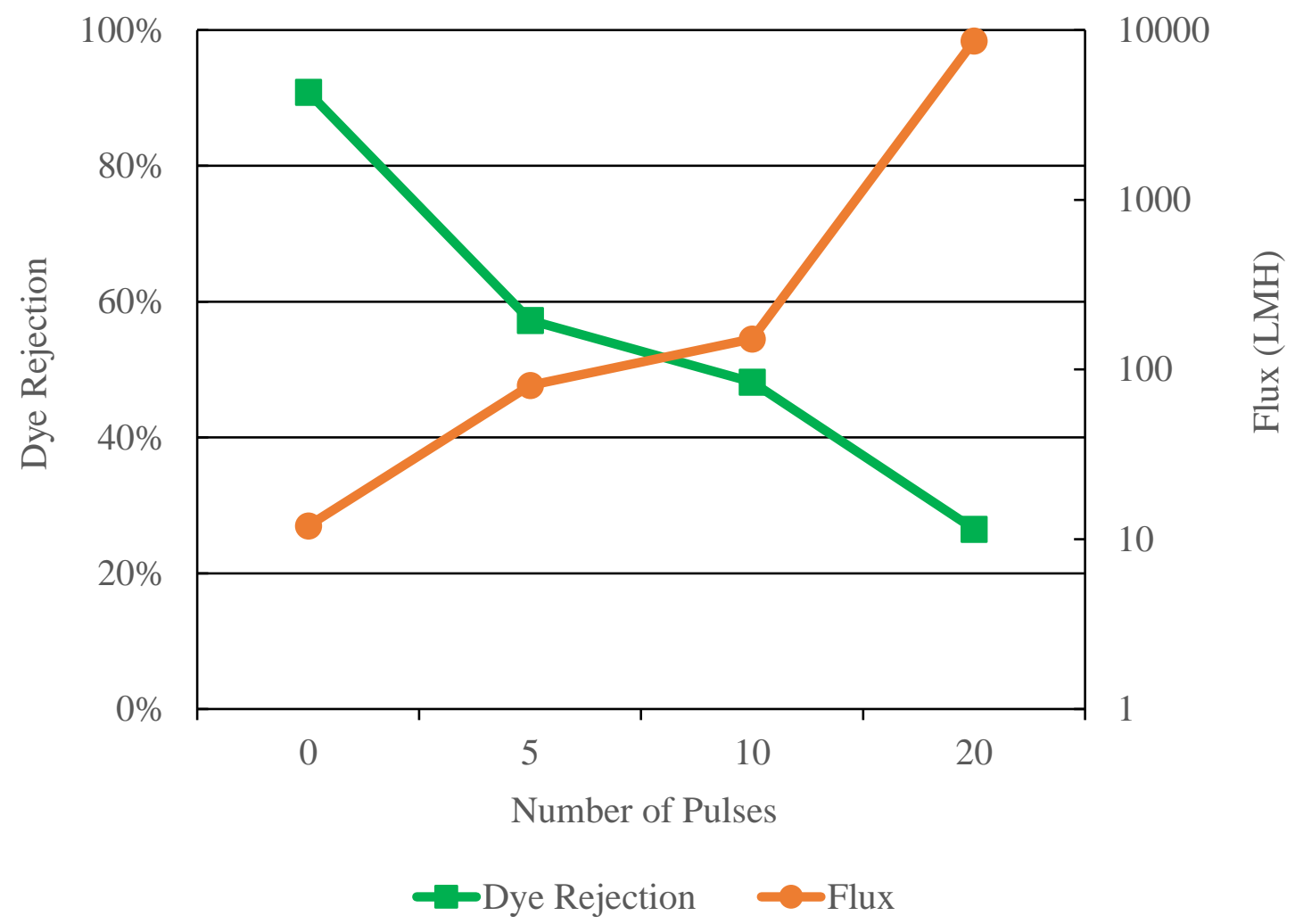

FIGURE 27. Flux and Dye Rejection for Reduced Graphene Oxide Membranes.

The dye rejection percentage decreased in a nearly linear trend, while the membrane flux increased logarithmically. Depending on the application of the membrane, a single membrane with very high rejection or multiple membranes with lower rejection but higher flux could be useful. The incremental changes produced by increasing numbers of light pulses provides strong control over the defect size and filtration performance of reduced graphene oxide membranes. 


\section{CONCLUSIONS}

This empirical investigation has revealed important information about graphene oxide production. First, flash reduction is a viable method for reducing graphene oxide. Also, film color can be used a proxy for the reduction state of a graphene oxide film. Finally, the filtration characteristics of a graphene oxide membrane can be tuned using flash reduction.

Flash reduction is a commercially scalable method for incrementally reducing graphene oxide. The light pulses caused significant chemical changes in the graphene oxide film as evidenced by the changes measured in optical characteristics and topography. The speed and versatility of flash reduction addresses some of the barriers that currently limit graphene oxide's use as a filter membrane. Additional work needs to be done to fully integrate graphene oxide reduction into a scalable roll-to-roll production process, but the speed and effectiveness of flash reduction is a significant step forward.

Correlating film color with reduction state is an important part of developing a reliable manufacturing process. Optical testing is already used in the filtration industry to detect large scale defects. However, for graphene oxide, the color of the film is tied closely to microscopic and nanoscale changes in the films structure. The color is dependent on the amount of flash reduction used and indicates the film's filtration characteristics. The ability to detect the filtration characteristics of a membrane without destructive testing could greatly enhance the quality control process for graphene oxide production.

Finally, the graphene oxide films produced by flash reduction can be tuned to filter at different size scales and fluxes by adjusting the number and power of light pulses 
used. As-deposited graphene oxide was observed to be highly impermeable, rejecting $90 \%$ of the dye in the filtered solution at a low flux. Treatment with light dramatically increased flux but reduced the dye rejection percentage. This tunable filtration behavior could be used to quickly manufacture customizable filter membranes with specialized filtration characteristics from a single deposited graphene oxide film.

The process of flash reduction allows precise control of the final graphene oxide membrane quality and commercially scalable production. Color checking provides a fast and reliable way to verify membrane quality, reducing the costs for producing a reliable graphene oxide membrane. Finally, the tunable characteristics of graphene oxide membranes open possibilities for many potential applications across the filtration industry. 


\section{FUTURE WORK}

This study has highlighted areas that require further investigation. Subsequent work in the field of flash reduction of graphene oxide should seek to better understand aspects of the flash reduction process. Future experiments using graphene oxide as a filtration membrane need to better understand the tunable characteristics of graphene oxide films.

In order to better understand the flash reduction of graphene oxide in air, elemental analysis of the film are needed to verify which oxygen groups are being removed or modified. X-ray photoelectron spectroscopy (XPS) would be a good method for these tests because of the thinness of the graphene oxide film. By providing a breakdown of the elements present along with their bond energies, XPS data would better show why the graphene oxide pore size changes during flash reduction.

Resistance atomic force microscopy (AFM) could also be useful in measuring the conductivity of graphene oxide after flash reduction. The bulk films tested in this investigation were found to have little change in electrical conductivity over as-deposited graphene oxide despite their change in color. More completely reduced graphene oxide experiences a dramatic increase in conductivity. AFM can measure the change in conductivity of individual graphene oxide flakes, which could enable it to measure changes in conductivity that cannot be detected by bulk film conductivity measurements.

Another way to better understand flash reduction would be to investigate the effect of an oxygenated atmosphere on the final graphene oxide film. Previous tests of flash reduction have often taken place in a hydrogen atmosphere to expedite the removal of oxygen groups and prevent oxygen recombination. Future testing would indicate 
whether reduction atmosphere has a significant effect on graphene oxide's filtration characteristics.

Future tests of graphene oxide as a filtration material would need to verify its long-term stability in various solvents, especially water. The changes in pore size could also be measured by testing the rejection fractions of different-sized solutes. The optimum thickness of the graphene oxide membrane should also be tested to determine the best combination of filtrate rejection and flux.

Looking forward, graphene oxide has the potential to disrupt the membrane filtration industry by reducing costs for desalination and allowing more precise filtering of polymer-incompatible solvents. Water is the world's most produced commodity by weight, and the filtration industry is growing in importance as fresh water becomes more scarce. Graphene oxide membranes may allow new sources of water to be tapped by future generations. 


\section{REFERENCES CITED}

Azizighannad, S., Mitra, S. (2018). "Stepwise Reduction of Graphene Oxide (GO) and Its Effects on Chemical and Colloidal Properties." Sci Rep 8(1): 10083.

Bennig, G. (1986). Atomic force microscope and method for imaging surfaces with atomic resolution. United States, International Business Machines Corp. US4724318A.

Choi, S.-J., Kim, S-J, Kim, I-D (2016). "Ultrafast optical reduction of graphene oxide sheets on colorless polyimide film for wearable chemical sensors." NPG Asia Materials 8(9): e315-e315.

Chun Li, G. S. (2012). "Three-dimensional graphene architectures." Nanoscale: 55495563.

Compton, O. C., Nguyen, S. T. (2010). "Graphene oxide, highly reduced graphene oxide, and graphene: versatile building blocks for carbon-based materials." Small 6(6): 711-723.

Cortes, S. (2010). Oxidation and Reduction in Organic Chemistry. U. o. T. a. Dallas.

Dikin, D. A., Stankovich, S., Zimney, E. J., Piner, R. D., Dommett, G. H., Evmenenko, G., Nguyen, S. T., Ruoff, R. S. (2007). "Preparation and characterization of graphene oxide paper." Nature 448(7152): 457-460.

Druffel, T., R. Dharmadasa, B. W. Lavery and K. Ankireddy (2018). "Intense pulsed light processing for photovoltaic manufacturing." Solar Energy Materials and Solar Cells 174: $359-369$.

Eluyemi, M. S., Eleruja, M. A., Adedeji, A. V., Olofinjana, B., Fasakin, O., Akinwunmi, O. O., Ilori, O. O., Famojuro, A. T., Ayinde, S. A., Ajayi, E. O. B. (2016). "Synthesis and Characterization of Graphene Oxide and Reduced Graphene Oxide Thin Films Deposited by Spray Pyrolysis Method." Graphene 05(03): 143-154. 
Feynman, R. (1963). The Origin of the Refractive Index. The Feynman Lectures. C. I. o. Technology. New York.

Gensheimer, J., Broaddus, Jack, Lin, Yu-Chien, Cao, Yan (2015). "Scalable Production of Reduced Graphene Oxide (rGO) from Graphite Oxide (GO)." Young Scientist Journal.

Graphenea. (2017). "Graphenea Highly Concentrated GO Datasheet." from https://cdn.shopify.com/s/files/1/0528/7349/files/Graphenea_Highly_Concentrated_GO_ Datasheet_201709.pdf?9479867757688917157.

Huang, X., Yin, Z., Wu, S., Qi, X., He, Q., Zhang, Q., Yan, Q., Boey, F., Zhang, H. (2011). "Graphene-based materials: synthesis, characterization, properties, and applications." Small 7(14): 1876-1902.

Hunt, I. (2019). sp2 hybridization. U. o. Calgary.

Hunt, I. (2019). sp3 hybridization. U. o. Calgary.

Johra, F. T., Lee, Jee-Wook, Jung, Woo-Gwang (2014). "Facile and safe graphene preparation on solution based platform." Journal of Industrial and Engineering Chemistry 20(5): 2883-2887.

Khan, Z. U., Kausar, Ayesha, Ullah, Hidayat, Badshah, Amin, Khan, Wasid U. (2016). "A review of graphene oxide, graphene buckypaper, and polymer/graphene composites: Properties and fabrication techniques." Journal of Plastic Film \& Sheeting 32(4): 336379.

Kinchin, G. H. (1953). "The electrical properties of graphite." Royal Society 217(1128).

Long Chen, J.-H. M., Xiaoxin Ma, Lin Zhang, Qiong Chen, Lina Chen, Ruiqin Peng, Pengchao Si, Jinkui Feng, Yanhui Li, Jun Lou, Lijie Ci (2018). "High performance 
graphene oxide nanofiltration membrane prepared by electrospraying for wastewater purification." Carbon 130: 487-494.

Lowe, S. E., Zhong, Yu Lin (2016). Challenges of Industrial-Scale Graphene Oxide Production. Graphene Oxide: 410-431.

Ma, J., Ping, D., Dong, X. (2017). "Recent Developments of Graphene Oxide-Based Membranes: A Review." Membranes (Basel) 7(3).

MacNeill, W., Choi, C. H., Chang, C. H., Malhotra, R. (2015). "On the self-damping nature of densification in photonic sintering of nanoparticles." Sci Rep 5: 14845.

NCBI (2012). Methyl red. National Center for Biotechnology Information.

NCBI (2018). Ethanol, National Center for Biotechnology Information.

NCBI (2018). Sucrose, National Center for Biotechnology Information.

Pei, L., Li, Yu-Feng (2017). "Rapid and efficient intense pulsed light reduction of graphene oxide inks for flexible printed electronics." RSC Advances 7(81): 51711-51720.

Pei, S., Cheng, Hui-Ming (2012). "The reduction of graphene oxide." Carbon 50(9): 3210-3228.

Pumera, M. (2013). "Electrochemistry of graphene, graphene oxide and other graphenoids: Review." Electrochemistry Communications 36: 14-18.

R. K. Joshi, P. C., F. C. Wang, V. G. Kravets, Y. Su, I. V. Grigorieva, H. A. Wu, A. K. Geim, R. R. Nair (2014). "Precise and Ultrafast Molecular Sieving Through Graphene Oxide Membranes." Science 343(6172): 752-754.

Stobinski, L., Lesiak, B., Malolepszy, A., Mazurkiewicz, M., Mierzwa, B., Zemek, J., Jiricek, P., Bieloshapka, I. (2014). "Graphene oxide and reduced graphene oxide studied 
by the XRD, TEM and electron spectroscopy methods." Journal of Electron Spectroscopy and Related Phenomena 195: 145-154.

Suk, J. W., Piner, Richard D., An, Jinho, Ruoff, Rodney S. (2010). "Mechanical Properties of Monolayer Graphene Oxide." American Chemical Society.

Tegou, E., Pseiropoulos G., Filippidou, M.K., Chatzandroulis S. (2016). "Lowtemperature thermal reduction of graphene oxide films in ambient atmosphere: Infra-red spectroscopic studies and gas sensing applications." Microelectronic Engineering 159: 146-150.

Thomas, H. R., Day, Stephen P., Woodruff, William E., Vallés, Cristina, Young, Robert J., Kinloch, Ian A., Morley, Gavin W., anna, John V., Wilson, Neil R., Rourke, Jonathan P. (2013). "Deoxygenation of Graphene Oxide: Reduction or Cleaning?" Chemistry of Materials 25(18): 3580-3588.

Wanbin Li , W. W., Zhanjun Li (2018). "Controlling Interlayer Spacing of Graphene Oxide Membranes by External Pressure Regulation." ACS Nano 12(9): 9309-9317.

Williams, R. (2010). Photonic Sintering for Printed Electronics Using Intense Pulsed Light. X. Corporation.

Wu, J. B., Lin, M. L., Cong, X., Liu, H. N., Tan, P. H. (2018). "Raman spectroscopy of graphene-based materials and its applications in related devices." Chem Soc Rev 47(5): 1822-1873.

Yeh, C. N., Raidongia, K., Shao, J., Yang, Q. H., Huang, J. (2014). "On the origin of the stability of graphene oxide membranes in water." Nat Chem 7(2): 166-170.

Zhang, J., Yang, H., Shen, G., Cheng, P., Zhang, J., Guo, S. (2010). "Reduction of graphene oxide via L-ascorbic acid." Chem Commun (Camb) 46(7): 1112-1114. 
Zunita, M., Makertiharta, I., Irawanti, R., Prasetya, N., Wenten, I. G. (2018). "Graphene Oxide- Inorganic Composite Membrane: A Review." IOP Conference Series: Materials Science and Engineering 395.

Çengel, Y. A., Ghajar, Afshin J. (2015). Heat and Mass Transfer Fundamentals and Applications. New York, NY, McGraw-Hill Education. 


\section{APPENDIX I. DIGITAL COLOR ANALYSIS DATA COLLECTED}

TABLE III lists the digital color measurements presented in FIGURE 16. The experimental methods used to collect this data are detailed in the Digital Color Measurement section of Results and Discussion.

TABLE III

RAW DATA FROM DIGITAL COLOR MEASUREMENT EXPERIMENT.

\begin{tabular}{|c|c|c|c|c|c|c|c|c|}
\hline Sample & $\begin{array}{c}\text { Run } \\
\text { Order }\end{array}$ & $\begin{array}{c}\text { Power } \\
\text { Level } \\
(\mathrm{kV})\end{array}$ & $\begin{array}{c}\text { Number } \\
\text { of } \\
\text { Pulses }\end{array}$ & $\begin{array}{c}\text { Measurement } \\
\text { Location }\end{array}$ & Intensity & Red & Green & Blue \\
\hline 1 & 69 & 1.8 & 10 & 1 & 483 & 160 & 168 & 155 \\
\hline 1 & 5 & 1.8 & 10 & 2 & 495 & 165 & 171 & 159 \\
\hline 1 & 117 & 1.8 & 10 & 3 & 501 & 166 & 174 & 161 \\
\hline 1 & 25 & 1.8 & 10 & 4 & 510 & 169 & 177 & 164 \\
\hline 13 & 75 & 1.8 & 10 & 1 & 507 & 168 & 176 & 163 \\
\hline 13 & 49 & 1.8 & 10 & 2 & 508 & 171 & 174 & 163 \\
\hline 13 & 99 & 1.8 & 10 & 3 & 490 & 163 & 171 & 156 \\
\hline 13 & 7 & 1.8 & 10 & 4 & 520 & 173 & 181 & 166 \\
\hline 25 & 59 & 1.8 & 10 & 1 & 539 & 176 & 186 & 177 \\
\hline 25 & 98 & 1.8 & 10 & 2 & 516 & 172 & 178 & 166 \\
\hline 25 & 51 & 1.8 & 10 & 3 & 528 & 175 & 183 & 170 \\
\hline 25 & 139 & 1.8 & 10 & 4 & 543 & 179 & 190 & 174 \\
\hline 2 & 10 & 1.8 & 40 & 1 & 494 & 165 & 172 & 157 \\
\hline 2 & 1 & 1.8 & 40 & 2 & 485 & 165 & 167 & 153 \\
\hline 2 & 114 & 1.8 & 40 & 3 & 479 & 161 & 168 & 150 \\
\hline 2 & 133 & 1.8 & 40 & 4 & 511 & 171 & 178 & 162 \\
\hline 14 & 32 & 1.8 & 40 & 1 & 490 & 164 & 170 & 156 \\
\hline 14 & 136 & 1.8 & 40 & 2 & 477 & 162 & 164 & 151 \\
\hline 14 & 108 & 1.8 & 40 & 3 & 463 & 158 & 161 & 144 \\
\hline 14 & 120 & 1.8 & 40 & 4 & 484 & 162 & 169 & 153 \\
\hline 26 & 23 & 1.8 & 40 & 1 & 488 & 162 & 169 & 157 \\
\hline 26 & 64 & 1.8 & 40 & 2 & 507 & 172 & 174 & 161 \\
\hline 26 & 79 & 1.8 & 40 & 3 & 499 & 167 & 174 & 158 \\
\hline 26 & 73 & 1.8 & 40 & 4 & 505 & 169 & 175 & 161 \\
\hline 3 & 141 & 1.8 & 70 & 1 & 487 & 166 & 169 & 152 \\
\hline & & & & & & & & \\
\hline
\end{tabular}




\begin{tabular}{|c|c|c|c|c|c|c|c|c|}
\hline Sample & $\begin{array}{c}\text { Run } \\
\text { Order }\end{array}$ & $\begin{array}{c}\text { Power } \\
\text { Level } \\
\text { (kV) }\end{array}$ & $\begin{array}{c}\text { Number } \\
\text { of } \\
\text { Pulses }\end{array}$ & $\begin{array}{c}\text { Measurement } \\
\text { Location }\end{array}$ & Intensity & Red & Green & Blue \\
\hline 3 & 28 & 1.8 & 70 & 2 & 456 & 156 & 157 & 143 \\
\hline 3 & 116 & 1.8 & 70 & 3 & 445 & 152 & 155 & 138 \\
\hline 3 & 47 & 1.8 & 70 & 4 & 448 & 153 & 156 & 139 \\
\hline 15 & 70 & 1.8 & 70 & 1 & 468 & 160 & 160 & 148 \\
\hline 15 & 109 & 1.8 & 70 & 2 & 450 & 154 & 155 & 141 \\
\hline 15 & 113 & 1.8 & 70 & 3 & 464 & 158 & 160 & 146 \\
\hline 15 & 132 & 1.8 & 70 & 4 & 479 & 163 & 166 & 150 \\
\hline 27 & 110 & 1.8 & 70 & 1 & 460 & 157 & 160 & 143 \\
\hline 27 & 26 & 1.8 & 70 & 2 & 448 & 154 & 155 & 139 \\
\hline 27 & 17 & 1.8 & 70 & 3 & 465 & 160 & 164 & 141 \\
\hline 27 & 87 & 1.8 & 70 & 4 & 453 & 156 & 159 & 138 \\
\hline 4 & 115 & 1.8 & 100 & 1 & 447 & 153 & 154 & 140 \\
\hline 4 & 19 & 1.8 & 100 & 2 & 430 & 149 & 147 & 134 \\
\hline 4 & 82 & 1.8 & 100 & 3 & 394 & 136 & 137 & 121 \\
\hline 4 & 89 & 1.8 & 100 & 4 & 463 & 158 & 161 & 144 \\
\hline 16 & 48 & 1.8 & 100 & 1 & 442 & 152 & 153 & 137 \\
\hline 16 & 2 & 1.8 & 100 & 2 & 456 & 156 & 157 & 143 \\
\hline 16 & 4 & 1.8 & 100 & 3 & 436 & 150 & 151 & 135 \\
\hline 16 & 60 & 1.8 & 100 & 4 & 442 & 153 & 155 & 134 \\
\hline 28 & 58 & 1.8 & 100 & 1 & 472 & 161 & 164 & 147 \\
\hline 28 & 55 & 1.8 & 100 & 2 & 431 & 150 & 148 & 133 \\
\hline 28 & 39 & 1.8 & 100 & 3 & 439 & 152 & 154 & 133 \\
\hline 28 & 72 & 1.8 & 100 & 4 & 469 & 162 & 164 & 143 \\
\hline 5 & 131 & 2 & 10 & 1 & 490 & 164 & 170 & 156 \\
\hline 5 & 125 & 2 & 10 & 2 & 483 & 163 & 167 & 153 \\
\hline 5 & 122 & 2 & 10 & 3 & 487 & 162 & 170 & 155 \\
\hline 5 & 121 & 2 & 10 & 4 & 511 & 170 & 178 & 163 \\
\hline 17 & 68 & 2 & 10 & 1 & 477 & 159 & 165 & 153 \\
\hline 17 & 140 & 2 & 10 & 2 & 470 & 159 & 161 & 150 \\
\hline 17 & 43 & 2 & 10 & 3 & 454 & 152 & 159 & 143 \\
\hline 17 & 105 & 2 & 10 & 4 & 472 & 158 & 164 & 150 \\
\hline 29 & 144 & 2 & 10 & 1 & 481 & 161 & 167 & 153 \\
\hline 29 & 38 & 2 & 10 & 2 & 486 & 165 & 167 & 154 \\
\hline 29 & 130 & 2 & 10 & 3 & 472 & 158 & 165 & 149 \\
\hline 29 & 11 & 2 & 10 & 4 & 490 & 163 & 171 & 156 \\
\hline 6 & 66 & 2 & 40 & 1 & 449 & 152 & 154 & 143 \\
\hline 6 & 100 & 2 & 40 & 2 & 444 & 152 & 152 & 140 \\
\hline & & & & & & & & \\
\hline
\end{tabular}




\begin{tabular}{|c|c|c|c|c|c|c|c|c|}
\hline Sample & $\begin{array}{c}\text { Run } \\
\text { Order }\end{array}$ & $\begin{array}{c}\text { Power } \\
\text { Level } \\
(\mathrm{kV})\end{array}$ & $\begin{array}{c}\text { Number } \\
\text { of } \\
\text { Pulses }\end{array}$ & $\begin{array}{c}\text { Measurement } \\
\text { Location }\end{array}$ & Intensity & Red & Green & Blue \\
\hline 6 & 56 & 2 & 40 & 3 & 452 & 154 & 156 & 142 \\
\hline 6 & 97 & 2 & 40 & 4 & 469 & 160 & 163 & 146 \\
\hline 18 & 44 & 2 & 40 & 1 & 461 & 157 & 159 & 145 \\
\hline 18 & 92 & 2 & 40 & 2 & 450 & 153 & 155 & 142 \\
\hline 18 & 36 & 2 & 40 & 3 & 466 & 159 & 162 & 145 \\
\hline 18 & 137 & 2 & 40 & 4 & 469 & 160 & 163 & 146 \\
\hline 30 & 61 & 2 & 40 & 1 & 465 & 158 & 160 & 147 \\
\hline 30 & 95 & 2 & 40 & 2 & 465 & 159 & 160 & 146 \\
\hline 30 & 84 & 2 & 40 & 3 & 454 & 155 & 158 & 141 \\
\hline 30 & 46 & 2 & 40 & 4 & 475 & 162 & 165 & 148 \\
\hline 7 & 52 & 2 & 70 & 1 & 438 & 150 & 150 & 138 \\
\hline 7 & 40 & 2 & 70 & 2 & 430 & 147 & 147 & 136 \\
\hline 7 & 13 & 2 & 70 & 3 & 421 & 145 & 146 & 130 \\
\hline 7 & 22 & 2 & 70 & 4 & 448 & 154 & 155 & 139 \\
\hline 19 & 78 & 2 & 70 & 1 & 461 & 157 & 159 & 145 \\
\hline 19 & 134 & 2 & 70 & 2 & 453 & 155 & 156 & 142 \\
\hline 19 & 86 & 2 & 70 & 3 & 439 & 152 & 154 & 133 \\
\hline 19 & 71 & 2 & 70 & 4 & 455 & 157 & 158 & 140 \\
\hline 31 & 83 & 2 & 70 & 1 & 461 & 157 & 159 & 145 \\
\hline 31 & 21 & 2 & 70 & 2 & 468 & 160 & 160 & 148 \\
\hline 31 & 74 & 2 & 70 & 3 & 438 & 151 & 153 & 134 \\
\hline 31 & 138 & 2 & 70 & 4 & 445 & 152 & 155 & 138 \\
\hline 8 & 77 & 2 & 100 & 1 & 462 & 158 & 159 & 145 \\
\hline 8 & 34 & 2 & 100 & 2 & 441 & 151 & 151 & 139 \\
\hline 8 & 53 & 2 & 100 & 3 & 436 & 150 & 151 & 135 \\
\hline 8 & 35 & 2 & 100 & 4 & 450 & 154 & 155 & 141 \\
\hline 20 & 102 & 2 & 100 & 1 & 414 & 142 & 142 & 130 \\
\hline 20 & 104 & 2 & 100 & 2 & 422 & 144 & 144 & 134 \\
\hline 20 & 9 & 2 & 100 & 3 & 400 & 138 & 139 & 123 \\
\hline 20 & 42 & 2 & 100 & 4 & 430 & 148 & 149 & 133 \\
\hline 32 & 37 & 2 & 100 & 1 & 432 & 147 & 149 & 136 \\
\hline 32 & 103 & 2 & 100 & 2 & 426 & 145 & 146 & 135 \\
\hline 32 & 29 & 2 & 100 & 3 & 412 & 141 & 144 & 127 \\
\hline 32 & 129 & 2 & 100 & 4 & 421 & 144 & 147 & 130 \\
\hline 9 & 65 & 2.2 & 10 & 1 & 480 & 160 & 166 & 154 \\
\hline 9 & 20 & 2.2 & 10 & 2 & 486 & 165 & 167 & 154 \\
\hline 9 & 12 & 2.2 & 10 & 3 & 463 & 155 & 162 & 146 \\
\hline & & & & & & & & \\
\hline
\end{tabular}




\begin{tabular}{|c|c|c|c|c|c|c|c|c|}
\hline Sample & $\begin{array}{c}\text { Run } \\
\text { Order }\end{array}$ & $\begin{array}{c}\text { Power } \\
\text { Level } \\
(\mathrm{kV})\end{array}$ & $\begin{array}{c}\text { Number } \\
\text { of } \\
\text { Pulses }\end{array}$ & $\begin{array}{c}\text { Measurement } \\
\text { Location }\end{array}$ & Intensity & Red & Green & Blue \\
\hline 9 & 45 & 2.2 & 10 & 4 & 484 & 162 & 168 & 154 \\
\hline 21 & 143 & 2.2 & 10 & 1 & 478 & 160 & 166 & 152 \\
\hline 21 & 62 & 2.2 & 10 & 2 & 475 & 161 & 163 & 151 \\
\hline 21 & 91 & 2.2 & 10 & 3 & 473 & 157 & 166 & 150 \\
\hline 21 & 111 & 2.2 & 10 & 4 & 470 & 157 & 164 & 149 \\
\hline 33 & 85 & 2.2 & 10 & 1 & 475 & 159 & 165 & 151 \\
\hline 33 & 123 & 2.2 & 10 & 2 & 505 & 169 & 175 & 161 \\
\hline 33 & 16 & 2.2 & 10 & 3 & 487 & 163 & 170 & 154 \\
\hline 33 & 107 & 2.2 & 10 & 4 & 502 & 167 & 176 & 159 \\
\hline 10 & 90 & 2.2 & 40 & 1 & 434 & 148 & 150 & 136 \\
\hline 10 & 27 & 2.2 & 40 & 2 & 431 & 147 & 147 & 137 \\
\hline 10 & 94 & 2.2 & 40 & 3 & 456 & 151 & 159 & 146 \\
\hline 10 & 57 & 2.2 & 40 & 4 & 436 & 150 & 151 & 135 \\
\hline 22 & 124 & 2.2 & 40 & 1 & 425 & 145 & 147 & 133 \\
\hline 22 & 126 & 2.2 & 40 & 2 & 422 & 144 & 144 & 134 \\
\hline 22 & 31 & 2.2 & 40 & 3 & 427 & 146 & 149 & 132 \\
\hline 22 & 33 & 2.2 & 40 & 4 & 454 & 155 & 158 & 141 \\
\hline 34 & 41 & 2.2 & 40 & 1 & 443 & 150 & 152 & 141 \\
\hline 34 & 30 & 2.2 & 40 & 2 & 437 & 148 & 150 & 139 \\
\hline 34 & 142 & 2.2 & 40 & 3 & 431 & 147 & 149 & 135 \\
\hline 34 & 106 & 2.2 & 40 & 4 & 458 & 156 & 158 & 144 \\
\hline 11 & 8 & 2.2 & 70 & 1 & 426 & 145 & 147 & 134 \\
\hline 11 & 128 & 2.2 & 70 & 2 & 442 & 150 & 150 & 142 \\
\hline 11 & 15 & 2.2 & 70 & 3 & 415 & 143 & 144 & 128 \\
\hline 11 & 76 & 2.2 & 70 & 4 & 439 & 151 & 152 & 136 \\
\hline 23 & 135 & 2.2 & 70 & 1 & 423 & 144 & 146 & 133 \\
\hline 23 & 24 & 2.2 & 70 & 2 & 405 & 137 & 138 & 130 \\
\hline 23 & 127 & 2.2 & 70 & 3 & 441 & 150 & 152 & 139 \\
\hline 23 & 80 & 2.2 & 70 & 4 & 435 & 148 & 150 & 137 \\
\hline 35 & 63 & 2.2 & 70 & 1 & 413 & 140 & 142 & 131 \\
\hline 35 & 3 & 2.2 & 70 & 2 & 395 & 135 & 135 & 125 \\
\hline 35 & 14 & 2.2 & 70 & 3 & 426 & 146 & 147 & 133 \\
\hline 35 & 88 & 2.2 & 70 & 4 & 429 & 146 & 148 & 135 \\
\hline & & & & & & & & \\
\hline
\end{tabular}




\begin{tabular}{|c|c|c|c|c|c|c|c|c|}
\hline Sample & $\begin{array}{c}\text { Run } \\
\text { Order }\end{array}$ & $\begin{array}{c}\text { Power } \\
\text { Level } \\
(\mathrm{kV})\end{array}$ & $\begin{array}{c}\text { Number } \\
\text { of } \\
\text { Pulses }\end{array}$ & $\begin{array}{c}\text { Measurement } \\
\text { Location }\end{array}$ & Intensity & Red & Green & Blue \\
\hline 12 & 101 & 2.2 & 100 & 1 & 429 & 146 & 148 & 135 \\
\hline 12 & 54 & 2.2 & 100 & 2 & 408 & 138 & 139 & 131 \\
\hline 12 & 67 & 2.2 & 100 & 3 & 417 & 143 & 144 & 130 \\
\hline 12 & 96 & 2.2 & 100 & 4 & 429 & 147 & 148 & 134 \\
\hline 24 & 112 & 2.2 & 100 & 1 & 417 & 143 & 144 & 130 \\
\hline 24 & 93 & 2.2 & 100 & 2 & 413 & 141 & 141 & 131 \\
\hline 24 & 118 & 2.2 & 100 & 3 & 405 & 139 & 140 & 126 \\
\hline 24 & 50 & 2.2 & 100 & 4 & 421 & 144 & 147 & 130 \\
\hline 36 & 81 & 2.2 & 100 & 1 & 401 & 137 & 137 & 127 \\
\hline 36 & 119 & 2.2 & 100 & 2 & 409 & 139 & 139 & 131 \\
\hline 36 & 6 & 2.2 & 100 & 3 & 396 & 136 & 137 & 123 \\
\hline 36 & 18 & 2.2 & 100 & 4 & 417 & 143 & 144 & 130 \\
\hline
\end{tabular}




\section{APPENDIX II. ELECTRICAL CONDUCTIVITY MEASUREMENTS}

To measure the reduction level of the graphene oxide films being tested, electrical conductivity measurements were made with using a four-point-probe attached to a Keithley multimeter and a two point probe attached to a Keithley picoammeter. The resulting measurements are displayed in TABLE IV.

TABLE IV.

REPRESENTATIVE MEASUREMENTS OF ELECTRICAL RESISTIVITY FOR GRAPHENE OXIDE AT VARIOUS STAGES OF REDUCTION

\begin{tabular}{|c|c|c|c|c|c|c|}
\hline \multirow{2}{*}{$\begin{array}{c}\text { Number } \\
\text { of } \\
\text { Pulses }\end{array}$} & \multicolumn{5}{|c|}{ Resistivity $(\mathrm{k} \Omega$ ) } & \multirow{2}{*}{$\begin{array}{c}\text { Average } \\
\text { Resistivity } \\
(\mathrm{k} \Omega)\end{array}$} \\
\hline & Meas. 1 & Meas. 2 & Meas. 3 & Meas. 4 & Meas. 5 & \\
\hline 0 & $76,000,000$ & $80,000,000$ & $82,000,000$ & $84,000,000$ & $75,000,000$ & $79,400,000$ \\
\hline 3 & $68,000,000$ & $65,000,000$ & $80,000,000$ & $80,000,000$ & $87,000,000$ & $76,000,000$ \\
\hline 6 & $57,000,000$ & $82,000,000$ & $86,000,000$ & $89,000,000$ & $85,000,000$ & $79,800,000$ \\
\hline 10 & 13.26 & 13.8 & 8.18 & 7.26 & 5.2 & 9.54 \\
\hline 15 & 8.75 & 4.57 & 6.65 & 3.18 & 4.55 & 5.54 \\
\hline
\end{tabular}

The graphene oxide films displayed very high resistivity in their as-deposited state. No significant change in resistivity was measured in response to a low number of xenon lamp pulses. When the number of pulses was increased and speckling appeared on the film, the resistivity of the film dramatically decreased. This change in resistivity indicated the transition to reduced graphene oxide (Pei 2012). 


\section{APPENDIX III. SUPPLEMENTAL FILTRATION STUDY}

In an attempt to further optimize the filtration characteristics of the graphene oxide membranes, another filtration study was run with samples reduced at $1.8 \mathrm{kV}$ pulse powers. The absorbance peaks, measured rejection fractions, and membrane fluxes are displayed in FIGURE 28, Table V, and Table VI.

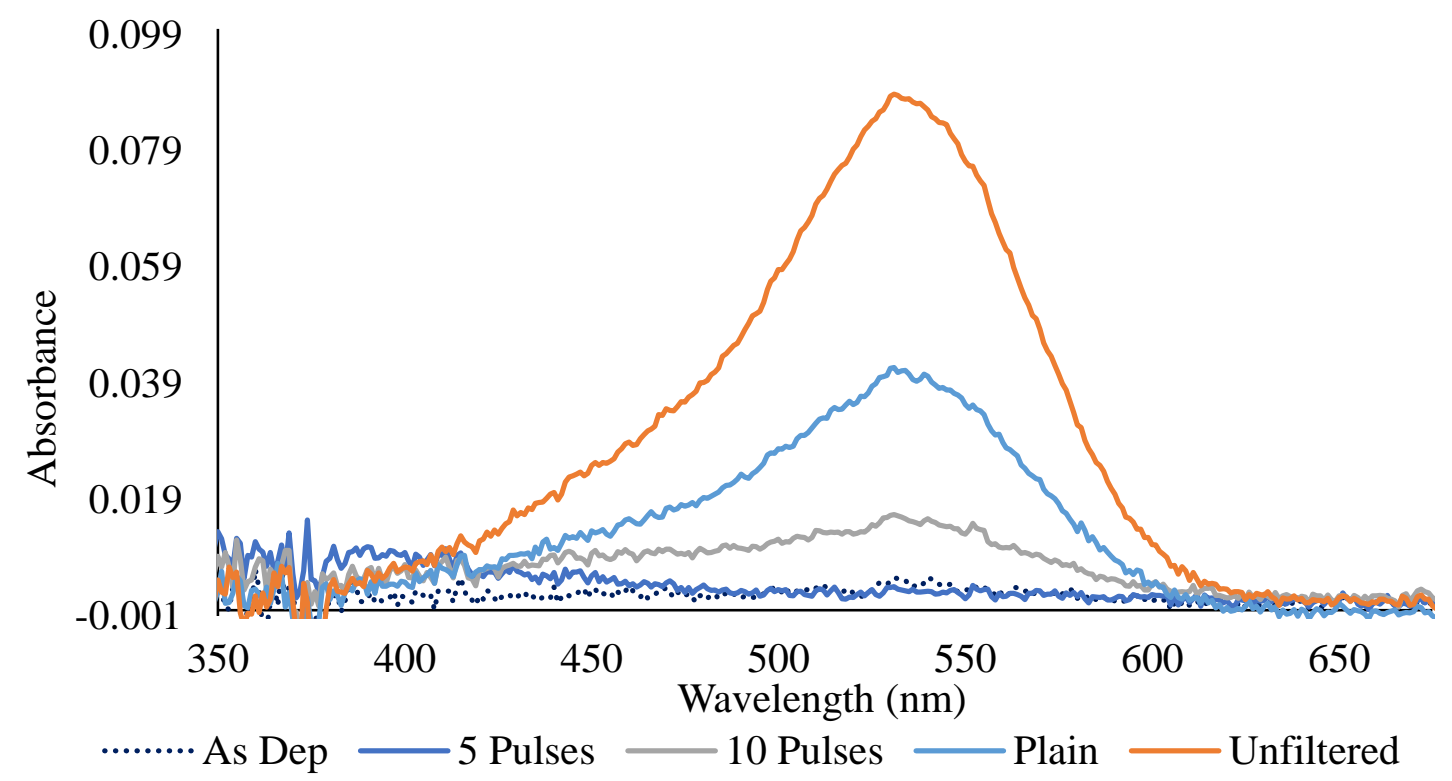

FIGURE 28. Filtered Solution Absorbance for 1.8 kV Pulsed Graphene Oxide. 
TABLE V.

\section{REJECTION FRACTIONS FOR GRAPHENE OXIDE FILMS REDUCED AT $1.8 \mathrm{KV}$ PULSE POWERS.}

\begin{tabular}{|c|c|c|}
\hline Sample Type & Peak Absorbance & Dye Rejection (\%) \\
\hline Unfiltered & 0.0582 & - \\
\hline Blank Substrate & 0.0325 & $53.4 \%$ \\
\hline As-deposited & 0.0053 & $94.2 \%$ \\
\hline 5 Pulses & 0.0249 & $88.1 \%$ \\
\hline 10 Pulses & 0.0301 & $81.9 \%$ \\
\hline
\end{tabular}

The graphene oxide films exposed to $1.8 \mathrm{kV}$ light pulses displayed higher rejection fractions and lower fluxes than the samples exposed to $2.2 \mathrm{kV}$. The $1.8 \mathrm{kV}$ samples showed a trend of higher fluxes and lower rejection fractions at greater numbers of pulses. This trend is similar to the behavior of the samples exposed to $2.2 \mathrm{kV}$.

TABLE VI

MEMBRANE FLUX FOR GRAPHENE OXIDE MEMBRANES EXPOSED TO $1.8 \mathrm{KV}$ LIGHT PULSES.

\begin{tabular}{|c|c|c|c|}
\hline Sample Type & Number of Pulses & Flow Time (min:s) & Flux LMH $\left(\frac{L}{m^{2} \cdot h r}\right)$ \\
\hline Blank Substrate & - & $00: 10$ & 4320 \\
\hline Graphene Oxide & 0 & $60: 00$ & 12.0 \\
\hline Graphene Oxide & 5 & $50: 57$ & 14.1 \\
\hline Graphene Oxide & 10 & $13: 37$ & 52.9 \\
\hline
\end{tabular}

The smaller changes in flux and reject fraction indicate that the lower power level provides more control for precisely tuning membrane properties. Further testing is required to determine the optimal number and power of pulses for specific membrane applications. 